\title{
Mechanisms Underlying the Early Establishment of Thalamocortical Connections in the Rat
}

\author{
Zoltán Molnár, Richard Adams, and Colin Blakemore \\ University Laboratory of Physiology, Oxford OX1 3PT, United Kingdom
}

\begin{abstract}
We labeled axonal projections using carbocyanine dyes in the developing rat brain to study cellular interactions that might underlie the establishment of thalamocortical connectivity. By embryonic day 14 (E14), groups of neurons in the ventral diencephalon and the primitive internal capsule have established projections to the dorsal thalamus, and thalamic fibers pass in topographic order among them. Simultaneously, axons from the early-born cells in both subplate and marginal zone (i.e., the original cortical preplate) establish an ordered array that fills the intermediate zone. Thalamic axons and preplate fibers meet in the lateral part of the internal capsule (at E15 for occipital cortex and dorsolateral thalamus). Subsequently, selective labeling of corresponding thalamic and early corticofugal projections reveals thalamic fibers growing in association with early corticofugal axons, right up to the cortical subplate. A small carbocyanine crystal implanted at any point in the cortex shortly after
\end{abstract}

the arrival of thalamic axons (E16 for the occipital cortex) labels a single, tight bundle containing both descending and ascending fibers, rather than two separate tracts, providing further evidence for intimate topographic association of the two axon systems. Crystals placed in a row, parasagittally or coronally along the hemisphere, reveal separate, topographically distributed, discrete fiber bundles throughout the pathway, leading to spatially ordered groups of back-labeled thalamic cells. These results indicate that the topography of thalamic axons is maintained throughout the pathway and that they reach the cortex by associating with the projections of a number of preexisting cells, including the preplate scaffold.

Key words: cortex; thalamus; rat; pioneer axons; development; subplate; perireticular nucleus; preplate; "handshake" hypothesis; internal capsule; thalamic reticular nucleus; axon guidance
The adult mammalian neocortex consists of numerous areas, differing in connectivity and functional properties (Peters and Jones, 1985), many of which have a distinctive cytoarchitectural appearance (Brodmann, 1909). Thalamic axons, the carriers of most afferent information to the cortex, arrive before the majority of cortical neurons are born (Lund and Mustari, 1977; Rakic, 1977; Wise and Jones, 1978; Shatz and Luskin, 1986). These early afferent projections, if they are topographically distributed and distinctive for each particular area, could play a part in initiating regional differentiation (O'Leary, 1989). In that case, unraveling the mechanisms by which thalamic axons are directed toward their target regions is important in understanding how cortical organization is determined.

The representation of the thalamus on the cortex and the topographic distribution of thalamic axons have been studied extensively in postnatal and adult rodents (Caviness and Frost, 1980; Frost and Caviness, 1980; Crandall and Caviness, 1984; Bernardo and Woolsey, 1987; Caviness, 1988; Agmon and Connors, 1991; Agmon et al., 1993, 1995; Mitrofanis and Guillery, 1993). All areas of the rodent neocortex receive a thalamic input. In general, neighborhood relationships are preserved in the pat-

Received March 3, 1998; revised May 8, 1998; accepted May 14, 1998.

This study was supported by grants from the Medical Research Council (MRC), the Wellcome Trust, the Soros-Hungarian Academy of Sciences Foundation, the Human Frontier Science Program, and Merton College (Oxford, UK). It forms part of the work of the Oxford McDonnell-Pew Centre and the MRC Interdisciplinary Research Centre for Cognitive Neuroscience, for which R.A. is a Research Scientist. Z.M. held an MRC Training Fellowship. We are indebted to Laurence Waters, Lorraine Chapell, and William Hinkes for help with photography and to Pat Owens and Duncan Fleming for excellent animal care.

Correspondence should be addressed to Zoltán Molnár, University Laboratory of Physiology, Parks Road, Oxford OX1 3PT, UK.

Copyright (C) 1998 Society for Neuroscience $\quad 0270-6474 / 98 / 185723-23 \$ 05.00 / 0$ tern of projection, but, in some cases, contiguous neocortical fields receive input from nonadjacent thalamic nuclei. However, studies of the mature projection do not directly illuminate its initial topography or the means by which connections are established. The spatial relationships of thalamic cell groups might change substantially, and thalamic axons themselves might undergo local rearrangement, after their projection to the cortex.

The capacity to trace axons in fixed embryonic tissue offered by the carbocyanine dye technique has stimulated many recent studies of early axonal outgrowth between cortex and thalamus for several species and various parts of the projection system (McConnell et al., 1989; Blakemore and Molnár, 1990; Molnár and Blakemore, 1990; Catalano et al., 1991, 1996; De Carlos and O'Leary, 1992; Erzurumlu and Jhaveri, 1992; Ghosh and Shatz, 1992; Miller et al., 1993; Clasca et al., 1995; Métin and Godement, 1996). However, no coherent picture has emerged of the relationships between growing thalamic axons and other fiber systems or of the mechanisms by which thalamic axons navigate to their appropriate target regions.

McConnell et al. (1989) drew attention to the fact that axons from early postmitotic neurons of the cortical subplate (MarinPadilla, 1971; Luskin and Shatz, 1985a,b) pioneer the pathway from the cortex toward subcortical targets, before neurons of layers 5 and 6 have migrated into position. They suggested that these early corticofugal axons might play a part in guiding subsequent descending and ascending projections.

Here we characterize the entire period of development of early corticofugal and thalamocortical projections in the rat and the degree of order that both sets of fibers maintain. We describe the cellular elements that thalamic axons encounter as they grow out from the diencephalon, and specifically we present evidence that 
they associate closely with axons emanating from early cortical neurons.

\section{MATERIALS AND METHODS}

Hooded Lister rats, whose gestation period is 21-22 d, were time-mated at the University Laboratory of Physiology (Oxford, UK). Assessment of the embryonic age of the fetuses was based on the plug date, defined as embryonic day 0 (E0). Fetuses were removed by cesarean section under pentobarbital anesthesia $(100 \mathrm{mg} / \mathrm{kg}$, i.p., to the pregnant female) at various stages, from the 13 th to the 21 st or 22 nd postconceptual day. They were immediately chilled on ice and transcardially perfused with $4 \%$ paraformaldehyde in $0.1 \mathrm{M}$ phosphate buffer, $\mathrm{pH} 7.4$, delivered through a glass micropipette under a dissecting microscope. Postnatal animals of various ages were anesthetized by chilling [up to postnatal day 4 (P4)], or by overdose of intraperitoneal sodium pentobarbital (if older than P4), before perfusion.

The brains were removed, and, after overnight post-fixation (in $4 \%$ buffered paraformaldehyde), single crystals $(0.1-0.3 \mathrm{~mm}$ diameter) of fluorescent carbocyanine dyes $\left[1,1^{\prime}\right.$-dioctadecyl 3,3,3'3'-tetramethylindocarbocyanine perchlorate (DiI), 4-[4-(dihexadecylamino)styryl]- $N$ methylpyridinium iodide (DiA), 4-[4-(didecylamino)styryl]- $N$-methylpyridinium iodide (DiAsp), and 3,3'-dipentyloxacarbocyanine iodide (DiO); Molecular Probes, Eugene, OR] were inserted into different parts of the cortex or diencephalon under an operating microscope (Godement et al., 1987).

For each cortical placement, a single carbocyanine crystal was inserted into a tiny slit made in the cortex with the tip of a pair of fine forceps or, in embryonic brains, with a thin stainless steel wire. The depth to which the crystal was inserted into the cortex varied with age, from as little as $0.1 \mathrm{~mm}$ at E13 to $0.5 \mathrm{~mm}$ in the oldest animals. In half of these brains, we made deposits of a number of different dyes, at points across the cortex, usually spaced 1-2 mm apart in either a parasagittal or a coronal row in one hemisphere. For most of these experiments, we used alternating placements of the two dyes that are best transported, DiI and DiA, which are clearly distinguishable under different wavelengths of fluorescent illumination.

For crystal placements in the thalamus [usually in the vicinity of the putative lateral geniculate nucleus (LGN)], we first transected the brainstem coronally, rostral to the superior colliculus, to expose the posterior, dorsal thalamus. For insertions into deeper or more rostral thalamic nuclei (e.g., the ventrobasal complex), we bisected the brain sagittally and cut away the medial part of the diencephalon to expose the relevant part of the thalamus. Then a single carbocyanine crystal was inserted into a small slit made in the tissue with the tip of a fine pair of forceps under an operating microscope.

In other specimens, the diencephalon was removed from the hemisphere by means of a parasagittal cut through the internal capsule, and a small crystal of carbocyanine dye was inserted into the exposed telencephalic face of the internal capsule, to stain descending connections reaching that region from other parts of the hemisphere, as well as any ascending axons that had already passed through the internal capsule. Table 1 lists the number of brains (both hemispheres) used for the various studies at each developmental stage (E13 to P8).

After crystal insertion, the brains were stored in fixative or in PBS containing $0.1 \% \mathrm{Na}$ azide, to prevent contamination, at room temperature $\left(22^{\circ} \mathrm{C}\right)$ or at $37^{\circ} \mathrm{C}$. They were incubated for periods ranging from 1 to 10 weeks, depending on age and temperature (Table 2), to allow diffusion of the dye. We came to prefer using room temperature because of the slightly lower background labeling. At the end of the incubation period the brains were embedded in $5 \%$ agar, and coronal or horizontal sections, $75-250 \mu \mathrm{m}$ thick, were cut on a vibratome (Oxford Instruments). All sections were counterstained with bisbenzimide (10 $\mathrm{min}$ in $2.5 \mu \mathrm{g} / \mathrm{ml}$ solution in PBS; Riedel-De Haèn AG, Seelze-Hannover, Germany) or acridine orange ( $10 \mu \mathrm{g} / \mathrm{ml}$ in PBS; Molecular Probes). The sections were coverslipped under Hydromount (National Diagnostics) or glycerol-phenylenediamine solution $(0.1 \%$ p-phenylenediamine, $10 \%$ PBS, and $90 \%$ glycerol, made up and stored according to the method of Johnson et al., 1982).

For the three-dimensional serial reconstructions, we coverslipped the sections under PBS, sealed the coverslips with Paraseal (Raymond A. Lamb, London, UK), and imaged the sections within $24 \mathrm{hr}$ to prevent differential shrinking and distortion of the individual members of the series. From the serial sections, double- or triple-exposure color micrographs were taken (Kodak Ektachrome 400 ASA color slide film), and
Table 1. Numbers of animals studied and dye placement sites

\begin{tabular}{lcccc} 
& \multicolumn{2}{l}{ Dye placements } & & \\
\cline { 2 - 5 } Age & Thalamus & Cortex & $\begin{array}{l}\text { Cortex and } \\
\text { thalamus }\end{array}$ & $\begin{array}{l}\text { Internal } \\
\text { capsule }\end{array}$ \\
\hline E13 & & 6 & & \\
E13.5 & & 6 & & 6 \\
E14 & 14 & 6 & & 6 \\
E14.5 & 5 & 4 & 8 & 14 \\
E15 & 17 & 5 & 10 & 16 \\
E15.5 & 8 & 4 & 8 & 12 \\
E16 & 8 & 12 & 8 & 6 \\
E18 & 6 & & & 6 \\
E19 & 6 & 7 & & 6 \\
E20 & 6 & 12 & & 6 \\
P0 & 8 & & & \\
P2 & 8 & 4 & & \\
P4 & 6 & & & \\
P8 & 6 & & & \\
\hline
\end{tabular}

E, Embryonic age (plug date taken as zero); P, postnatal age (day of birth, equivalent to E21-E22, taken as zero). At early embryonic ages, specimens fixed during the second half of the day are listed separately (E13.5, E14.5, and E15.5) from those taken during the first $12 \mathrm{hr}$ of the day (E13, E14, and E15).

\begin{tabular}{lll}
\hline Table 2. Carbocyanine dye incubation periods in weeks & \\
& \multicolumn{2}{l}{ Incubation temperature } \\
\cline { 2 - 3 } Age & $22^{\circ} \mathrm{C}$ & $37^{\circ} \mathrm{C}$ \\
\hline E13-E14.5 & 2 & 1 \\
E14.5-E16 & $3-6$ & $2-4$ \\
E18-P0 & 6 & 4 \\
P0-P8 & $6-10$ & $4-8$ \\
\hline
\end{tabular}

camera lucida drawings were made under epifluorescent illumination, using different filters to reveal the various dyes.

In early experiments, some sections from each brain (and from other control brains not used for dye tracing) were subsequently stained with cresyl violet to help with identification of the major divisions of the thalamus and to reveal the full thickness and lamination pattern of the cortex. Counterstaining with chromatin stains (bisbenzimide and acridine orange) helped us interpret the sections by revealing the outlines and boundaries of obvious cell groups (e.g., cortical layer I and layers in the hippocampus). Observation of nuclear chromatin staining with high-power objectives and/or obtaining fine optical sections with confocal microscopy enabled us to confirm whether labeled profiles were cell bodies.

Some sections, selected in conventional fluorescence microscopy, were subsequently examined and photographed with a laser-scanning confocal microscope (CLSM-Fluovert; Leica, Heidelberg, Germany). Digitized fine optical sections (up to 64 sections) were stacked to generate highresolution extended focus images (see Fig. $10 F$ ). Stereo pairs (with $\pm 7^{\circ}$ disparity) could be constructed from these three-dimensional data sets (see Fig. 10G).

\section{RESULTS}

This study is based on a total of 285 axon-tracing experiments, covering the period from E13, before the arrival of the first cells in the cerebral cortex, to P8, when the organization and innervation of the cortex appear virtually fully mature (see Table 1). For the sake of clarity we present the results in five parts:

(1) We describe the timing of outgrowth of thalamocortical fibers, their pattern of organization and initial topography, their arrival at the cortex, and invasion of the cortical plate, based on anterograde labeling from one or more dye crystals implanted in the dorsal thalamus. 

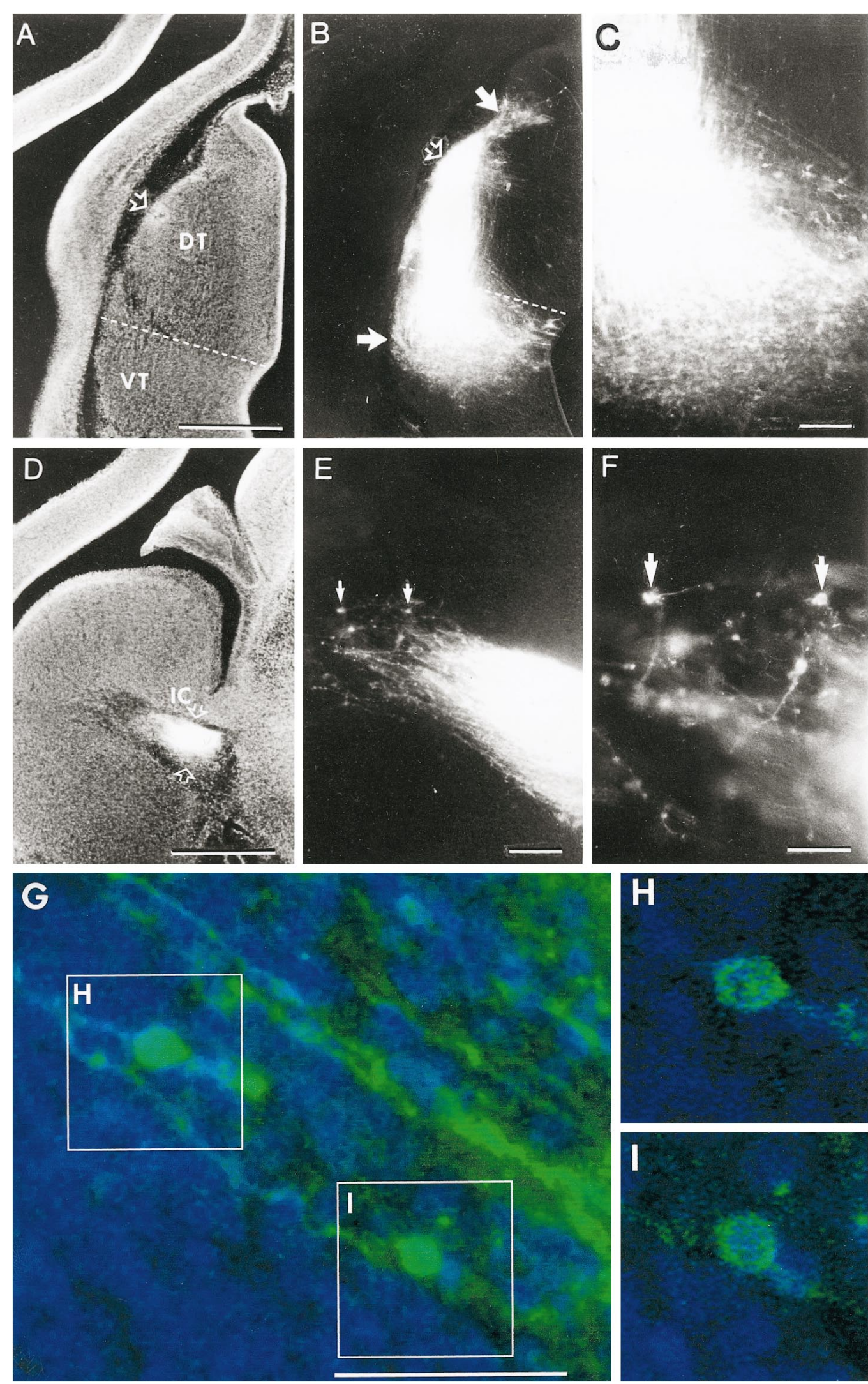

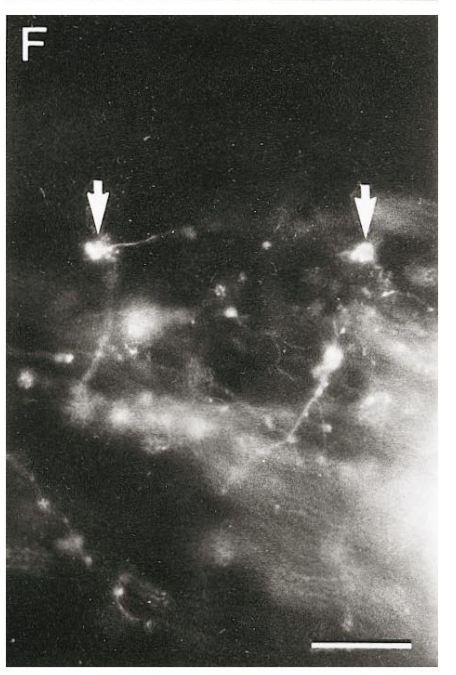

H

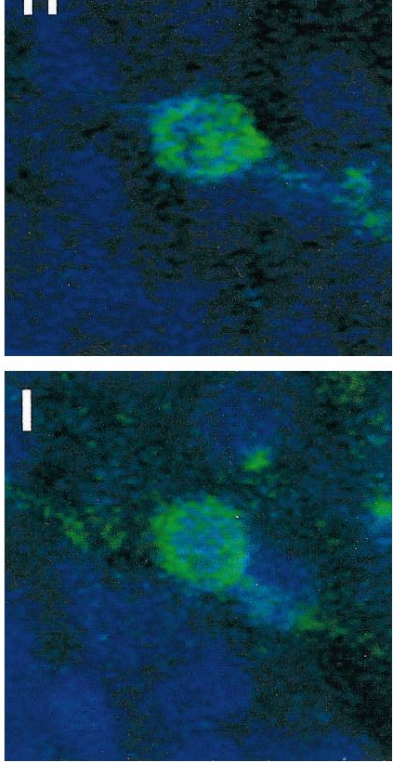

Figure 1. A small crystal of DiI was implanted into the dorsolateral part of the left thalamus of an embryonic rat brain fixed during the first half of E14. After 4 weeks incubation at room temperature, $100-\mu \mathrm{m}$-thick coronal sections were cut, counterstained with bisbenzimide, and examined by both fluorescence and laser-scanning confocal microscopy. $A$, Bisbenzimide staining of a coronal section shows the crystal implantation site (unfilled arrow) in the putative dorsal LGN. The interrupted line indicates the border between dorsal thalamus (DT) and ventral thalamus $(V T) . B$, DiI labeling in the same field as $A$. The labeled fiber bundle extends from the dorsal thalamus to the ventral diencephalon. There are numerous backlabeled cells within the thalamic reticular nucleus of the ventral thalamus ( filled arrow below the implantation site, which is indicated by an unfilled arrow) and a few above, in the epithalamus (filled arrow above). The mass of axons descending from the dorsal thalamus is presumably a mixture of the ascending axons of thalamic reticular cells and orthogradely labeled thalamofugal fibers from the presumptive LGN. $C$, High-power view of back-labeled thalamic reticular cells within the region indicated by the lower filled arrow in $B$. D, Two sections away from $A, 200 \mu \mathrm{m}$ anterior, where geniculofugal labeling reaches its full lateral extent, within the primitive internal capsule $(I C)$, beneath the medial ganglionic eminence. This double exposure shows both bisbenzimide-stained cells and DiIlabeled axons. $E, F$, Medium- and high-power views in the region of the fiber endings reveal a small number of back-labeled cells within the primitive internal capsule (filled arrows), a region called the perireticular nucleus by Mitrofanis (1992). $G$, An image from the same region of the internal capsule as $E$, showing both chromatin (blue) and DiI staining (green). Two stacked sets of three $2-\mu \mathrm{m}$-thick confocal sections, taken at identical optical planes but with different filters, have been combined. Many cell bodies (blue chromatin staining) are not backlabeled, but some certainly are. Two examples (outlined) are shown in higher power in $H$ and $I$, which are single confocal sections through the middle of the thickness of the cell bodies, in which the blue chromatin is clearly visible, surrounded by DiI staining. Scale bars: $A, B, D, 500$ $\mu \mathrm{m} ; C, E, 100 \mu \mathrm{m} ; F-H, 50 \mu \mathrm{m}$. 

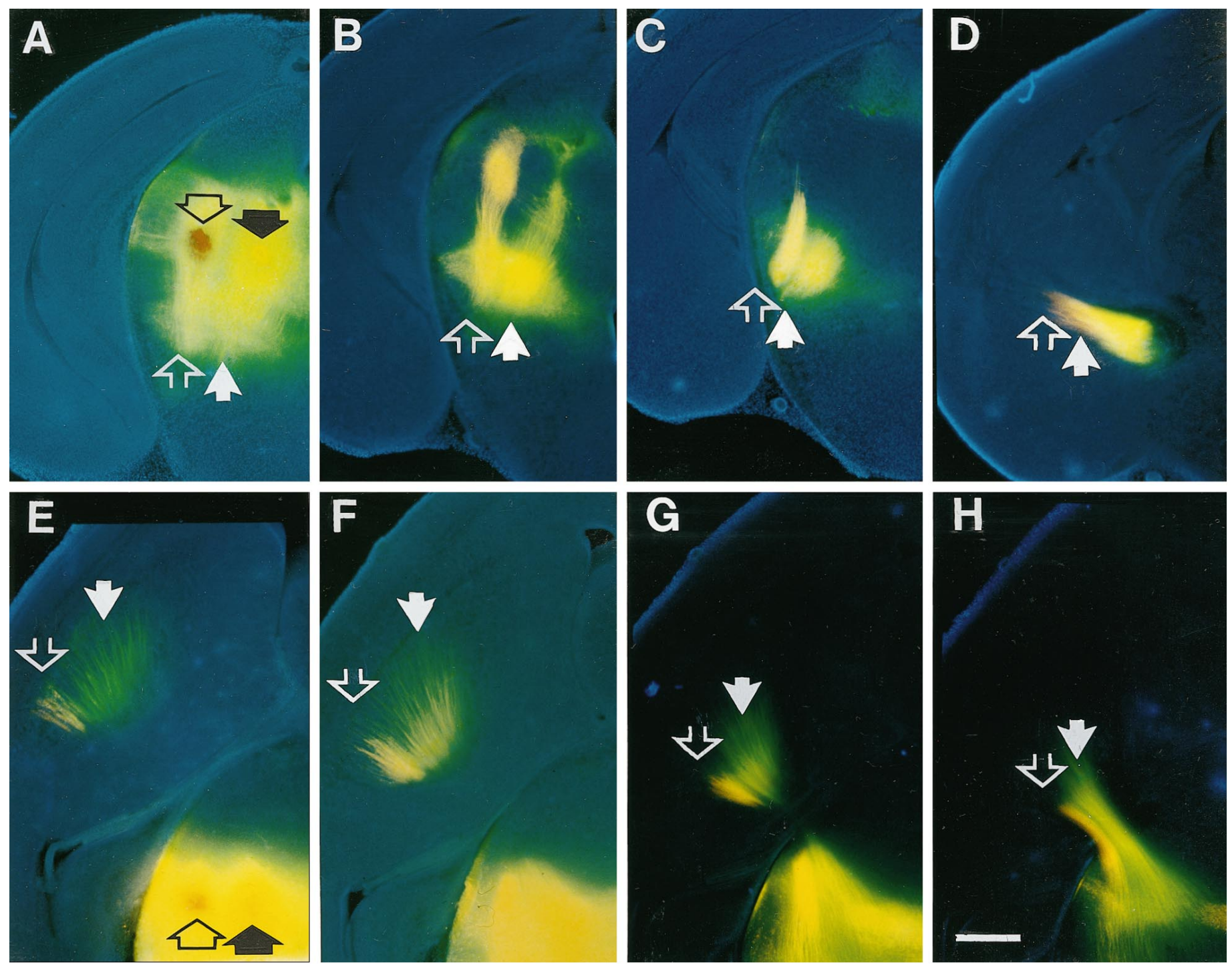

Figure 2. One crystal of DiI and one of DiA were inserted about $200 \mu \mathrm{m}$ apart (DiI more lateral) into the left dorsal thalamus of two E15 brains. After 3 weeks incubation at room temperature, one of the brains was sectioned coronally $(100 \mu \mathrm{m}$ thick) $(A-D)$, the other was sectioned horizontally $(E-H)$. The sections were counterstained with bisbenzimide. Fluorescence micrographs were taken by exposing the film three times to reveal the three different fluorescent signals. The serial coronal sections are presented in caudorostral sequence $(A-D)$, the horizontal sections dorsoventrally $(E-H)$. $A$, Crystal placement sites in dorsal thalamus. The dense cores, presumably corresponding to the major uptake sites, are indicated by black-outlined arrowheads ( filled, DiA; unfilled, DiI). They are surrounded by large, overlapping halos of dye. $B, C$, The two labeled bundles of thalamic fibers appear largely segregated as they descend in the ventral thalamus (white arrows, unfilled for DiI, filled for DiA). Groups of neurons back-labeled with DiI and DiA are visible in the thalamic reticular nucleus of the ventral thalamus, below the implantation sites (especially in $B$ ). $D$, At this level, rostal to $C$, the growing tips of the axons are seen within the primitive internal capsule. Here the two bundles largely overlap each other, but examination of neighboring sections shows that they are still segregated in the parasagittal plane, those from the more lateral thalamic site lying more caudal and heading for a more posterior region of the hemisphere. $E, F$, A similarly labeled brain was sectioned horizontally, to demonstrate the rostrocaudal separation of the two bundles of labeled thalamic fibers as they pass into the internal capsule $(H)$ and up toward the intermediate zone $(G, F, E)$. The fibers labeled with DiI (unfilled white arrows) from the more lateral thalamic implantation (unfilled black arrow in $E$ ) head to a more posterior region then those labeled with DiA ( filled arrows) from the more medial crystal placement ( filled black arrow in E). Scale bar, $500 \mu \mathrm{m}$.

(2) The cells of origin of the earliest corticofugal projections were labeled and examined after placement of crystals in the primitive internal capsule before thalamic fibers approach the cortex.

(3) The timing of outgrowth and topography of these early corticofugal projections is then described, on the basis of anterograde tracing from dye crystals placed in the cortex, before the arrival of thalamic axons.

(4) We then describe the relationship between early corticofugal and thalamocortical fibers-at their meeting point in the primitive internal capsule, within the intermediate zone, and as the thalamic axons approach the cortical subplate (from E14.5 to E16 for different parts of the cortex). This is based partly on double labeling (with different dyes placed in corresponding regions of thalamus and cortex) and partly on the labeling of both sets of axons and cortical cell bodies from crystals placed in the internal capsule.

(5) Finally, single- and multiple-crystal placements in the cortex, shortly after the arrival of thalamic axons, provided further information about the relationship between afferent and efferent 

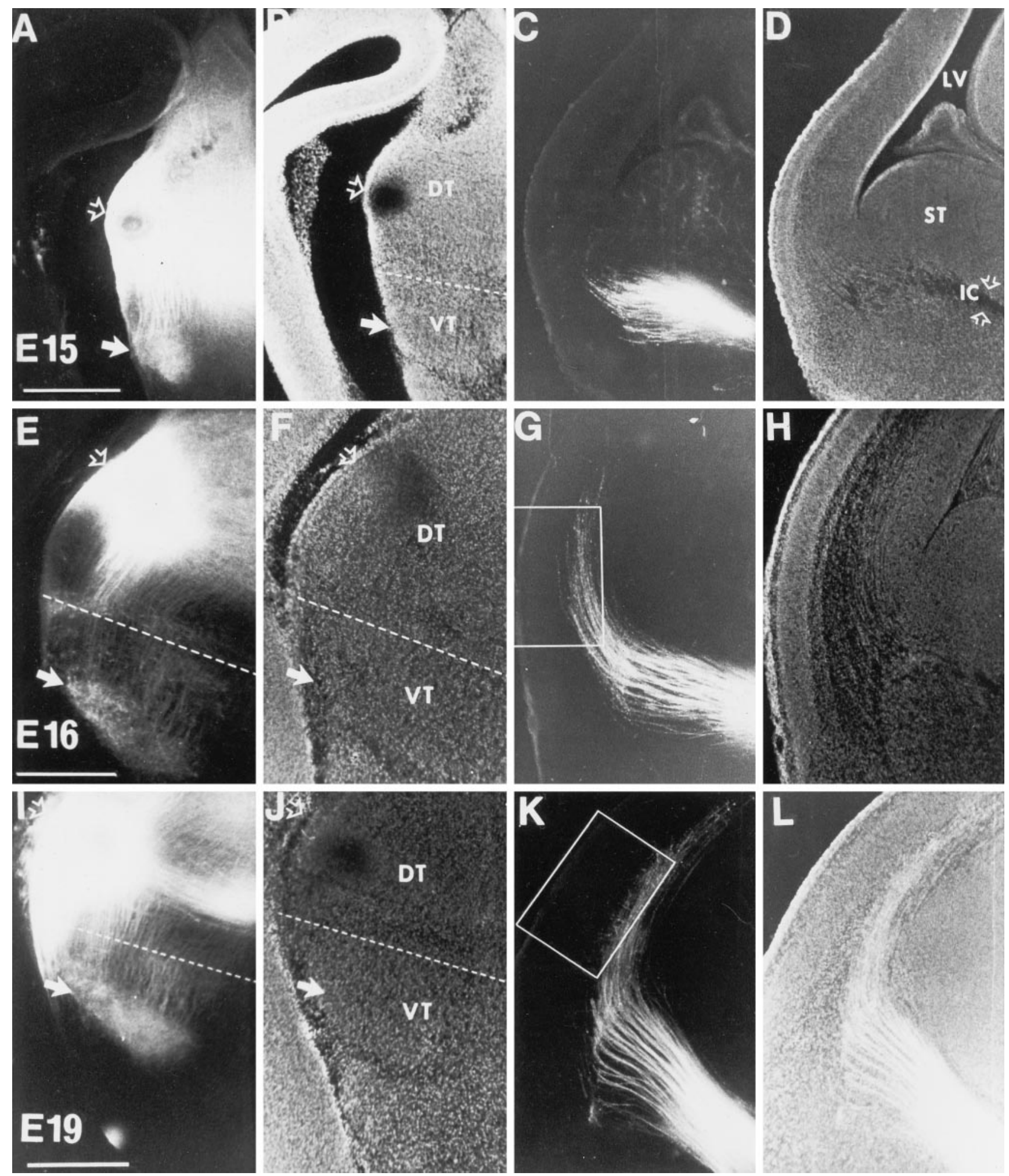

Figure 3. The advance of the thalamocortical fibers toward the cortex is revealed with a crystal placement into the putative LGN in the left dorsal thalamus $(D T)$ of brains at E15 (top row, $A-D$ ), E16 (middle row, $E-H$ ) and E19 (bottom row, $I-L$ ). After $2-6$ weeks incubation at $37^{\circ} \mathrm{C}, 100 \mu \mathrm{m}$ coronal sections were cut and counterstained with bisbenzimide. For each row, the first two panels $(A, E$, I, DiI staining; $B, F, J$, bisbenzimide counterstain) demonstrate the thalamic crystal implantation sites (unfilled arrows). Numerous cells are labeled in the thalamic reticular nucleus ( filled arrows) of the ventral thalamus $(V T)$. The right pairs of panels show the tips of the thalamic fibers at a more rostral level $(C, G, K, \mathrm{DiI} ; D, H, L$, bisbenzimide). The thalamic fibers are growing into the intermediate zone of the ventral telencephalon at E15. By E16, thalamic fibers have extended up into the convexity of the occipital cortex and reached the subplate layer. Even by E19 $(K)$ there is very little advance into the cortical plate itself. Note that no cells of the cortex are back-labeled from the dorsal thalamus at these stages. Higher-power photomicrographs of the regions indicated by white outline boxes in $G$ and $K$ are presented in Figure 5. $L V$, Lateral ventricle; $S T$, anlage of the corpus striatum; $I C$, primitive internal capsule, demarcated by unfilled arrows in $D$. Scale bar, $500 \mu \mathrm{m}$ for all panels. 


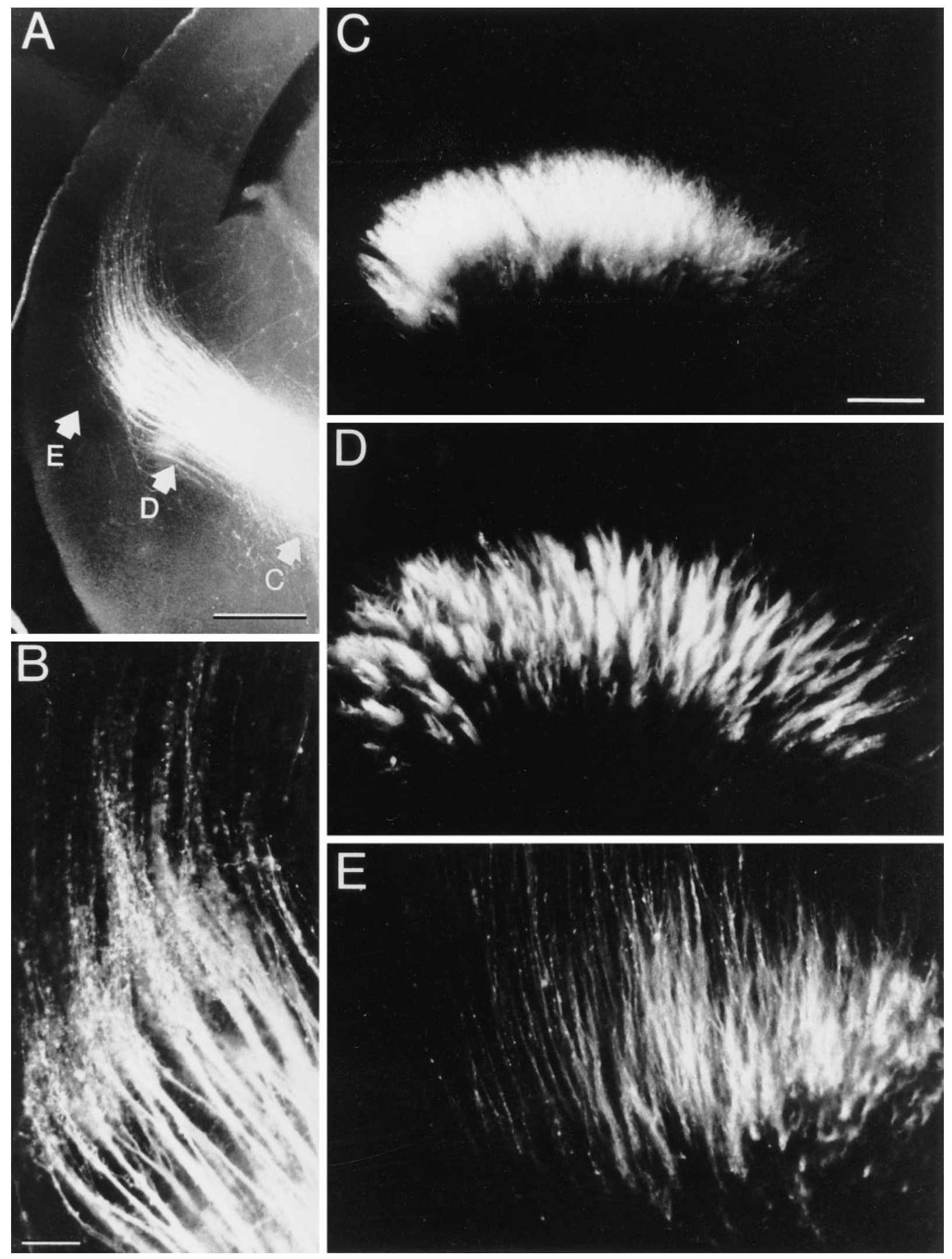

Figure 4. Thalamocortical axons show distinct patterns of organization at different points along their path to the cortex. Crystal placement into the left dorsal thalamus labels fibers that reach the intermediate zone beneath the ventrolateral cortex at E15.5 ( $0.5 \mathrm{~d}$ before they reach the occipital pole itself). $A$, Leaving the diencephalon, the fibers form fascicles, which open up in a fan-shaped manner. Viewed here in a coronal section, the order of the fibers is such that the inferior-lateral fascicles are destined for the more ventrolateral part of the cortex, whereas the superior-medial fascicles turn upward and head toward more dorsal cortical areas. The trajectories of these fascicles remain approximately parallel as they turn up into the intermediate zone; they do not cross each other to any significant degree. B. Higher-power view of the region where the fascicles break up into (Figure legend continues) 
axons and about the global topography of the pathway established between thalamus and cortex.

\section{Development of thalamocortical projections}

\section{Thalamocortical fibers grow out in topographic order among} cell groups that project to the thalamus

Implantation of a small crystal of carbocyanine dye into the dorsolateral part of the thalamus (including the presumptive LGN) on E14 reveals anterogradely labeled thalamic fibers that have already grown down through the diencephalon and are passing through the primitive internal capsule. The example in Figure 1 is representative of all 14 experiments performed at this age. The thalamic axons run under and through the anlage of the developing corpus striatum to reach the intermediate zone of the ventrolateral telencephalon at approximately E15.5 (see Fig. 3).

DiI implantation in the dorsal thalamus, even early on E14, also labels numerous cell bodies in the ventral diencephalon (presumably in the thalamic reticular nucleus; Mitrofanis and Baker, 1993) and a few within the primitive internal capsule, lying below both medial and lateral ganglionic eminences (Fig. $1 E, F$ ), in the region that Mitrofanis (1992) calls the perireticular nucleus. Even where they are close to the tips of growing thalamocortical axons, the back-labeled perireticular cells can be convincingly distinguished from the surrounding growth cones by their chromatin content, revealed by matching fine confocal optical sections collected with different filters to distinguish the carbocyanine dye and the bisbenzimide and/or acridine orange counterstaining (Fig. 1G,H,L). The fact that the cells of the thalamic reticular and perireticular nuclei are densely stained with carbocyanine dye (even after the shortest incubation periods) and are intermingled with entirely unlabeled cells makes us confident that they were labeled retrogradely along axons already projecting into the dorsal thalamus at E14, rather than by transcellular diffusion of dye from labeled thalamic axons.

We obtained some information about the topography of the initial outgrowth of thalamic fibers by tracing the axons labeled by two or more crystals of different dyes, placed in the dorsal thalamus. The entire thalamus is so small at this stage that these placements necessarily had to be very close together. However, such experiments reveal that fiber bundles originating from different thalamic regions remain separate as they descend in the ventral thalamus, enter the internal capsule, and grow through and under the developing striatum (Fig. 2). The location of the back-labeled thalamic reticular and perireticular cells was also clearly dependent on the location of the crystal placement within the dorsal thalamus, indicating at least some degree of topographic order in the projection of those two cell groups to the thalamus at very early stages.

The examples in Figure 2 show serial coronal sections (Fig. $2 A-D$ ) and horizontal (Fig. $2 E-H$ ) sections from two brains in which a pair of different carbocyanine dye crystals were placed into the dorsal thalamus, only $\sim 200 \mu \mathrm{m}$ apart in the coronal plane, at E15. The diffuse halos of dye around the individual crystals are large and overlapping, but it is likely that uptake of dye was largely restricted to the dense cores (Fig. 2A,E). In coronal sections, two differently colored bundles of axons are seen descending side-by-side through the ventral diencephalon (Fig. $2 A-C$ ). In the most rostral coronal section (Fig. $2 D$ ) the advancing wave front of thalamic fibers is passing through the primitive internal capsule, beneath the anlage of the striatum. Here, the two axon bundles overlap each other in the coronal plane, but the series of horizontally cut sections from a similarly labeled brain (Fig. $2 E-H$ ) shows that they are still quite separate in that plane. Fibers from the more medial thalamic site run rostral to those from the more lateral implantation. This suggests that the array of fibers from this region of the dorsal thalamus rotates by $\sim 90^{\circ}$ but maintains its topographic order as it leaves the diencephalon. Additional evidence for both the topography of the axon array and its rotation comes from retrograde labeling of the same axons from the cortex $\sim 1 \mathrm{~d}$ later (see below).

\section{Growth through the primitive internal capsule and intermediate zone}

Between E15 and E16, thalamocortical fibers pass through the primitive internal capsule (Fig. 3). The homogeneous, topographic array of axons leaving the diencephalon (Fig. 4C) parcellates into distinct fascicles, which open up in a fan-shaped pattern (Fig. 4D), as they pass within the anlage of the corpus striatum. The fibers do not appear to cross each other extensively at any point along their course, although some individual axons and small axon bundles can be seen switching from one fascicle to another within the striatal anlage (Fig. 4B). Viewed in coronal sections, the ordering of fibers is such that the inferior lateral fascicles are destined for the ventral part of the cortex, whereas the superior medial fascicles turn upward and head toward more dorsal cortical areas (Fig. 3G,K).

At the border between the corpus striatum and the intermediate zone the thalamic fibers defasciculate and form a fairly uniform array of individual fibers (Fig. $4 E$ ). The trajectories of the axons remain more or less parallel as they turn up into the intermediate zone. Fibers from the dorsolateral thalamus (primordial LGN) are advancing through the intermediate zone beneath the ventrolateral cortex at approximately E15.5, and they reach the subplate layer of the occipital cortex itself at E16 (Figs. 3-5 are examples representative of all experiments; see Table 1). As with the fascicles from which they emerge, the ordering of the individual fibers is such that the more inferior and lateral ones are destined for the more ventral cortical segment, whereas the more medial axons, situated deeper in the white matter, head toward more dorsal cortical segments (Figs. 3, 5). The entire, ordered array of thalamic fibers fills the full depth of the intermediate zone, from the cortical subplate down to the subventricular zone (Fig. 3G,H).

\section{Accumulation of thalamic fibers below the cortex}

Although axons from the region of the LGN reach the occipital cortex at approximately E16, they do not substantially invade the cortical plate for some time. Most of the labeled fibers

\section{$\leftarrow$}

individual fibers as they enter the intermediate zone. To examine the topography of fibers in more detail, the contralateral hemisphere (with an identical crystal placement to that in $A$ ) was cut perpendicular to the fiber path. $C, D$, $E$, Sections at levels corresponding to the labeled arrows in $A$. $C$, The fibers leave the diencephalon through the primitive internal capsule. The bundle is at its narrowest at this point. No fasciculation is apparent in this region. $D$, As the fibers run under the anlage of the corpus striatum, they form 30- to 50- $\mu \mathrm{m}$-thick fascicles, slightly separated from each other. The labeled fiber array loosens up and expands slightly (compare with $C$ ). $E$, The fiber bundles defasciculate as they reach the intermediate zone and turn under the cortical plate. The right side of the section still shows some fascicles, whereas the left demonstrates defasciculated fibers running approximately parallel to each other in the intermediate zone. Scale bars: $A, 300 \mu \mathrm{m} ; B, 50 \mu \mathrm{m} ; C-E, 100 \mu \mathrm{m}$. 

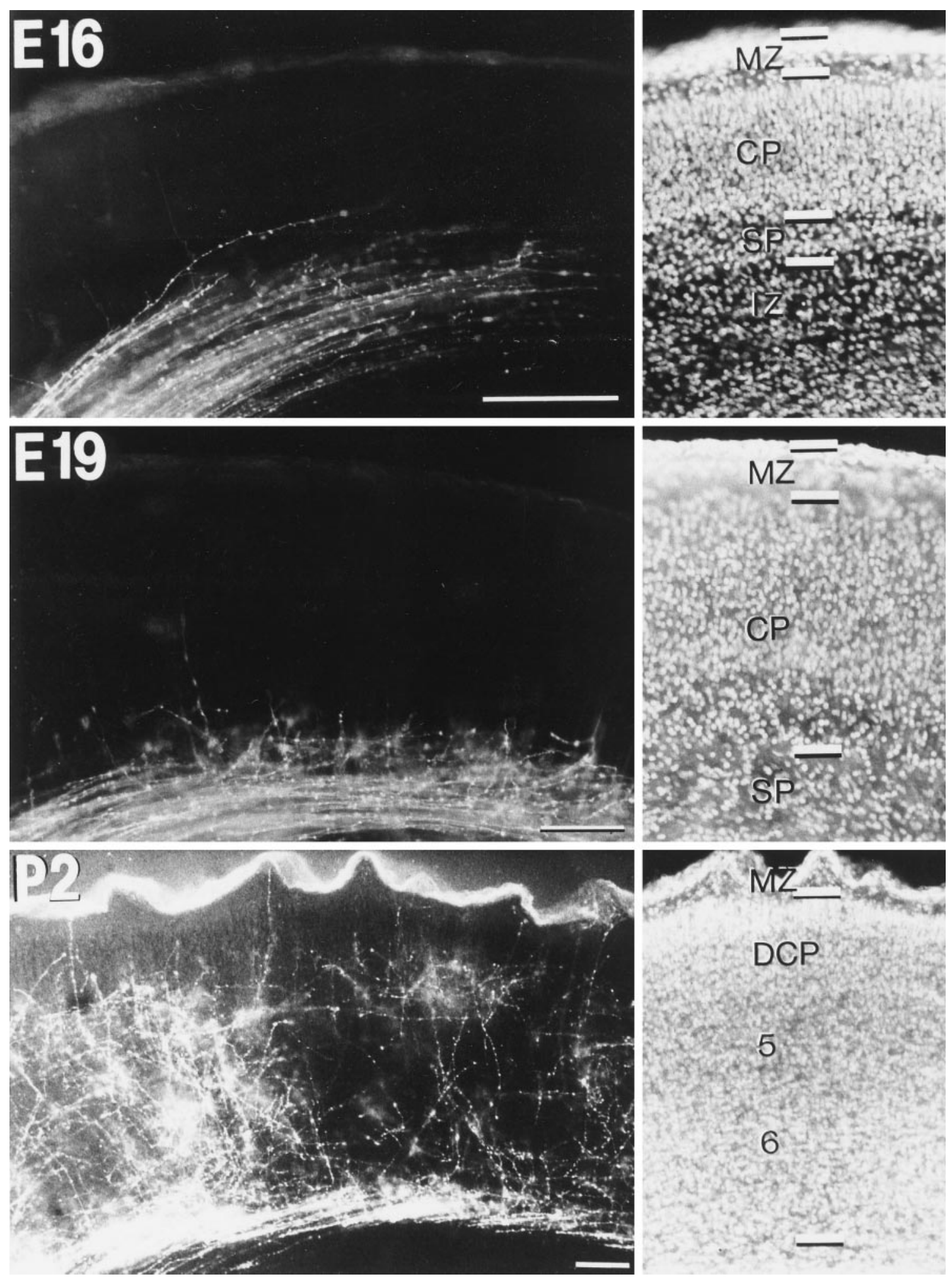

Figure 5. Three stages of thalamic fiber growth in the cortical target region of the left hemisphere, revealed by crystal placement in the dorsal thalamus: E16 (top row), E19 (middle row), and P2 (bottom row). Left panels, DiI labeling; right panels, bisbenzimide labeling of the same section. The examples shown for $E 16$ and $E 19$ are higher-power fluorescence photomicrographs from Figure 3, $G$ and $H$ and $K$ and $L$, respectively. The bottom row demonstrates fiber labeling in the cortex of a P2 animal after a similar crystal placement. Thalamic fibers reach the occipital cortex at E16 but remain mainly restricted to the subplate $(S P)$ and intermediate zone $(I Z)$. At $E 19$ some fibers have developed side branches, but the vast majority still lie within the SP and have not yet entered the cortical plate $(C P)$. By $P 2$, thalamic axons have turned up into the cortical plate en masse. They take irregular courses through layers 6 and 5, and the majority branch and terminate in presumptive layer 4, $\sim 300 \mu \mathrm{m}$ below the pial surface, directly underneath the dense cortical plate $(D C P)$, which consists of newly arrived, densely packed immature neurons. A few axons are seen extending up to the marginal zone $(M Z)$. Note that, with discrete crystal placements restricted to the dorsolateral thalamus, very few if any cell bodies are back-labeled in the cortical plate or the subplate, even at E19 and P2. Scale bar, $100 \mu \mathrm{m}$ for all panels. 

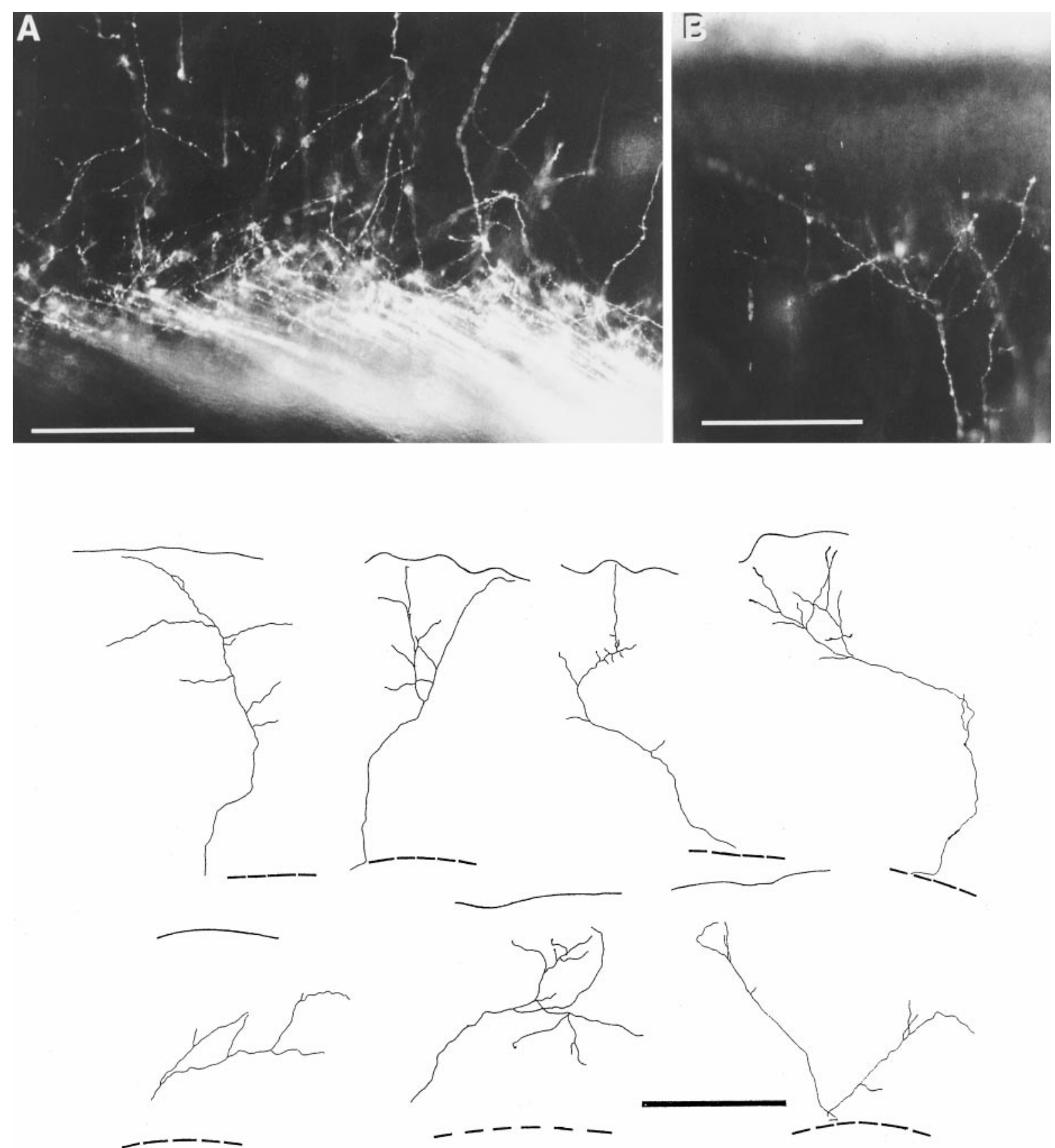

Figure 6. Photomicrographs and camera lucida drawings of thalamic fibers entering the right occipital cortex of a P2 rat labeled from a DiI crystal placed in the dorsal thalamus. $A$, The thalamic fibers form a remarkably parallel array until they enter the cortical plate, where they diverge and follow more irregular patterns, arborizing as they ascend to the middle layers. The fibers projecting to more lateral areas (right side) run superficial to those destined for more dorsal areas. The more ventrolateral cortex is innervated earlier, and the topographic ordering of fibers is reflected in the chronological sequence of their arrival under the cerebral cortex. $B$, The photomicrograph was taken from the same section as $A$ but focusing on a single arbor extending over a large area, some $200 \mu \mathrm{m}$ below the pial surface. To illustrate the variety of individual thalamic fiber arbors in the occipital cortex, just after they have entered the cortex, camera lucida drawings were made of individual arbors seen in $200-\mu \mathrm{m}$-thick coronal sections of P2 cortex. In each drawing the top continuous line marks the pial surface, whereas the interrupted line below shows the upper boundary of the white matter. It is quite likely that many individual arborizations extended outside the thickness of the $200 \mu \mathrm{m}$ section, because some arbors extend as far as $500 \mu \mathrm{m}$ within the plane of the section. Scale bars: $A, B, 200 \mu \mathrm{m}$; camera lucida drawings, $500 \mu \mathrm{m}$.

accumulate in and remain restricted to the intermediate and subplate zones until E19. During this period, thalamic fibers develop increasing numbers of local branches along their length within the subplate layer. These branches are nearly all directed upward toward the cortical plate, but most are short and restricted to the subplate region, at least in the occipital cortex. A few penetrate the lowest part of the cortical plate (Fig. 5, E19), and very rarely some are seen reaching close to the lower boundary of the dense cortical plate-the tightly packed, recently arrived, immature neurons that form the upper part of the cortical plate.

The generation of cortical plate neurons in the ventricular and 

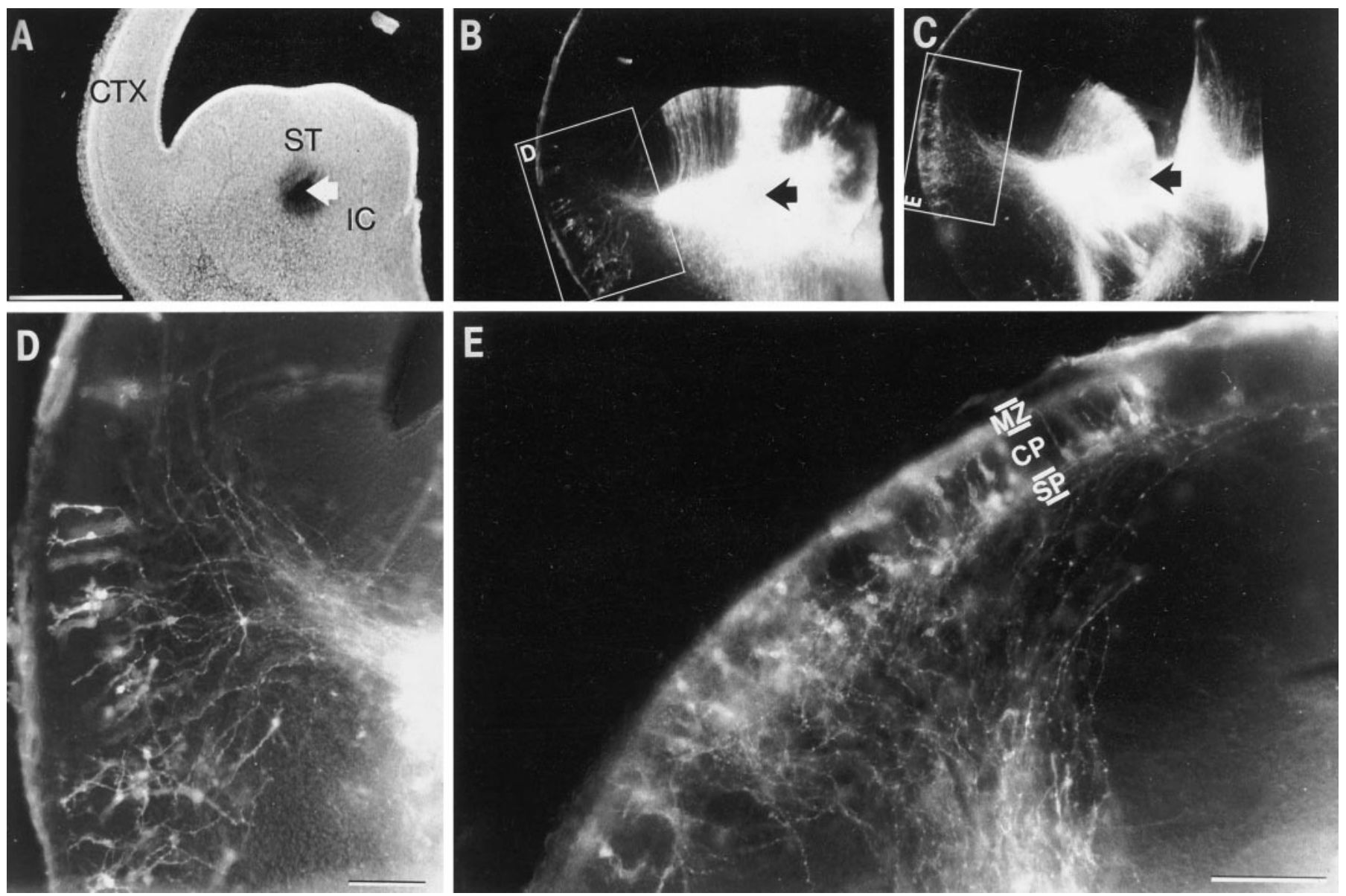

Figure 7. Results of implanting a DiI crystal into the primitive internal capsule on the left side at E14 $(A, B, D)$ and E14.5 $(C, E)$, showing bisbenzimide $(A)$ and DiI $(B-E)$ labeling. $A, B$, Matched images show the crystal placement (arrows) into the primitive internal capsule (IC). Numerous presumed radial glia are stained in the striatal anlage $(S T)$, and back-labeled cells are seen in perirhinal cortex and adjacent lateral neocortex. A large group of cells was also labeled within the primitive internal capsule itself (data not shown). $C$, DiI labeling in a matched section from a similar experiment in an E14.5 fetus. Neurons are now also back-labeled further dorsally in the cerebral wall. $D$, A high-power view of the lateral part of $B$ (indicated by the outline box) demonstrates back-labeled neurons, lying mainly at the base of the cortical plate in presumptive perirhinal cortex. Note that some of the cells in perirhinal cortex are pyramidal in form, with processes extending up toward the pial surface. Their morphology is quite different from the polygonal and polymorphic (presumed preplate) cells labeled in the lateral cortex (seen in $C, E$ ). $E$, Segment of cortex containing back-labeled cells (viewed with higher magnification from the outline box indicated in $C$ ). The majority of back-labeled cells in lateral neocortex are situated in the subplate and marginal zones, but there are also a few within the emerging cortical plate. $C T X$, Cortex; $M Z$, marginal zone; $C P$, cortical plate; $S P$, subplate. Scale bars: $A-C, 500 \mu$ m; $D, E, 100 \mu \mathrm{m}$.

subventricular zones is at its peak when thalamic axons arrive (Miller, 1988). Migrating cells therefore have to pass through the mass of fibers accumulating in the subplate layer, as well as between the subplate cells themselves.

\section{Invasion of the cortex}

Before approximately E19-E20, very few thalamic axons appear to have grown into the cortical plate itself in the occipital region (Fig. 5). Invasion on a massive scale suddenly begins at approximately E19-E20, just a couple of days before birth. The initial course taken by these invading fibers is distinctive and highly ordered; most make virtually a $90^{\circ}$ turn from their trajectory in the white matter, and the vast majority initially grow radially, straight up into the cortex. A small proportion take more erratic initial routes, running somewhat obliquely upwards (Fig. 6). The ingrowth could partly originate from the side branches of thalamic axons, some of which penetrate the cortical plate at earlier stages.

In favorable preparations, at all stages from E15 onward, indi- vidual thalamic axons can be seen forming an impressively ordered, parallel array running up through the intermediate zone and into the subplate layer below the cortex (Figs. 3G, 5, 6A). Fibers heading for more ventral and lateral cortex run superficial to those destined for more dorsal segments. Because the earlier axons enter ventral cortical areas first, and these areas develop relatively early, the ordering of thalamic fibers mirrors the temporal order of maturation of their cortical target areas.

\section{Establishment of laminar termination patterns}

By birth (P0, equivalent to E21-E22) and afterward, the trajectories of thalamic fibers within the cortical plate appear far less regular and parallel than in the white matter (Figs. 5, 6A). Some individual thalamic axons have branches in layer 6 of the cortical plate and may terminate there; but most of them branch and appear to terminate above in putative layer 5 and especially in layer 4. By approximately P2, most axons have bifurcated at least once near the top of layer 5 and have arborized quite widely in the lamina that is differentiating into layer 4 , directly below the 

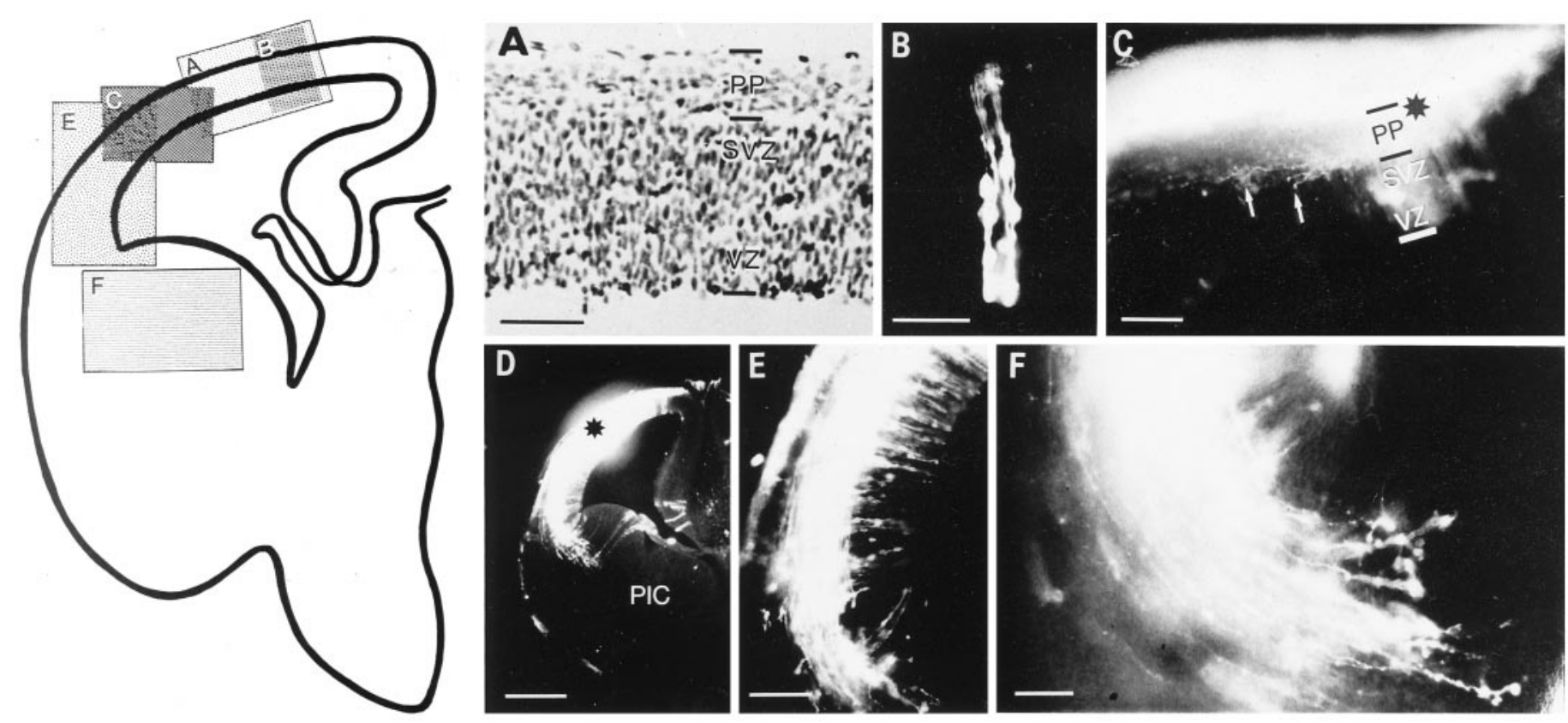

Figure 8. Outgrowth of the first corticofugal projections. The photomicrographs were taken from the regions indicated in the schematic drawing on the left: $A$ and $B$ at E13; $C$ at E13.5; and $D-F$ at E14.5. $A$, High-power photomicrograph taken from a 10- $\mu$ m-thick, cresyl-violet-stained coronal section of the occipital cortex of an E13 embryo. At this early stage the wall of the telencephalic vesicle consists of proliferative ventricular zone ( $V Z)$ and subventricular zone $(S V Z)$, with a thin layer of postmitotic cells beginning to accumulate to form the primordial plexiform zone or preplate ( $P P$ ). $B$, Coronal section of the cerebral wall at E13 (slightly less mature than $A$ ), stained with a small crystal of DiI inserted into the cortex nearby, leading to intense local labeling of a few radial processes, presumably of glia and/or germinal cells. At this very early stage, no cell bodies or any axons are seen at the outer edge of the cerebral wall, but a little later $(C-E)$, a dense plexus of axons grows out from the preplate. $C$, A crystal of DiI inserted, at the point indicated by an asterisk, in the convexity of the left hemisphere at E13.5 reveals the very first axons (arrows) arising from the thin layer of postmitotic cells forming the preplate $(P P)$, between the heavily stained pial surface and the subventricular zone. Radial processes are seen, out of the plane of focus, below the placement site. $D$, An E14.5 brain with a similar cortical DiI crystal placement (asterisk) reveals early corticof ugal axons descending and turning medially into the primitive internal capsule $(P I C)$. Individual cells and their processes are difficult to resolve by ordinary fluorescence microscopy because of the intensity of labeling of the somata and the dense plexus of fibers. $E$, Higher magnification of $D$. In dorsal regions, where the preplate is very thin, fibers are seen coursing among the early postmitotic cells. When the preplate is split by the emerging cortical plate (first in more ventral regions), most descending fibers are seen to belong to cells of the lower, subplate zone, but some originate from neurons of the marginal zone. When the crystal placement site is in the most ventral region of neocortex or in the perirhinal cortex, occasional back-labeled cells are seen in the primitive internal capsule, within the bundle of labeled axons. This suggests that fibers originating from more dorsal cortex are purely corticof ugal, whereas those from perirhinal cortex consist of both anterogradely labeled corticof ugal fibers and retrogradely labeled axons of "perireticular" cells lying within the primitive internal capsule. $F$, In higher power, the labeled fiber bundle from occipital cortex at E14.5 is seen entering the primitive internal capsule. These fibers end in large and elaborate growth cones. Scale bars: $A-C, F, 50 \mu \mathrm{m} ; D, 350 \mu \mathrm{m} ; E, 100 \mu \mathrm{m}$.

dense cortical plate, which is itself reducing in thickness as the upper layers mature. Many axons now appear to have lost their growth cones. A few fibers continue to grow all the way to the marginal zone, but the vast majority terminate within a fairly narrow laminar range and avoid the uppermost sector of the cortex.

Individual labeled axons were followed in $200-\mu \mathrm{m}$-thick sections and drawn via a camera lucida (Fig. 6). At P2 their arborizations in presumptive layer 4 typically extend over an area $\sim 300$ $\mu \mathrm{m}$ wide. This wide lateral spread of the terminal arbors of thalamic axons contrasts with the remarkable order with which they are arranged at the junction between white and gray matter (Figs. 5, 6A). Between P2 and approximately P8 (when the cortex has achieved its mature lamination), layer 4 and the arborizations of thalamic afferents within it appear to be progressively displaced downward as the supragranular layers thicken.

\section{Tracing from the internal capsule}

Through the entire period of outgrowth of thalamocortical fibers, their accumulation below the cortex and the initial invasion of the cortical plate, small crystal placements in the thalamus itself back-labeled very few cortical cells, even within the subplate, and none was ever labeled from crystals restricted to the dorsal thalamus (Figs. 3-6). Clasca et al. (1995) described similar findings in the ferret; they suggested that early corticof ugal projections from the subplate and layer 6 are delayed for several weeks in the cerebral white matter, and that axons from layer 5 are the first to pass through the internal capsule into the diencephalon. We used small crystal placements within the internal capsule itself to determine when corticofugal projections reach the internal capsule in the rat and which cortical neurons project through it at early stages.

At E14 a small crystal implanted into the primitive internal capsule back-labels numerous cell bodies and their processes within the presumptive perirhinal and lateral cerebral cortex. It also labels a segment of the striatal radial glia above and below the implantation site and some adjacent neurons within the internal capsule itself. Figure 7 demonstrates two representative examples from six experiments. Many of the back-labeled cells within the putative perirhinal cortex have a pyramidal shape and differ from the typical polymorphous, polygonal morphology of the labeled preplate cells in the lateral and dorsal cortex (Fig. 7, compare $D, E$, respectively). Even by E14-E14.5, in the more 

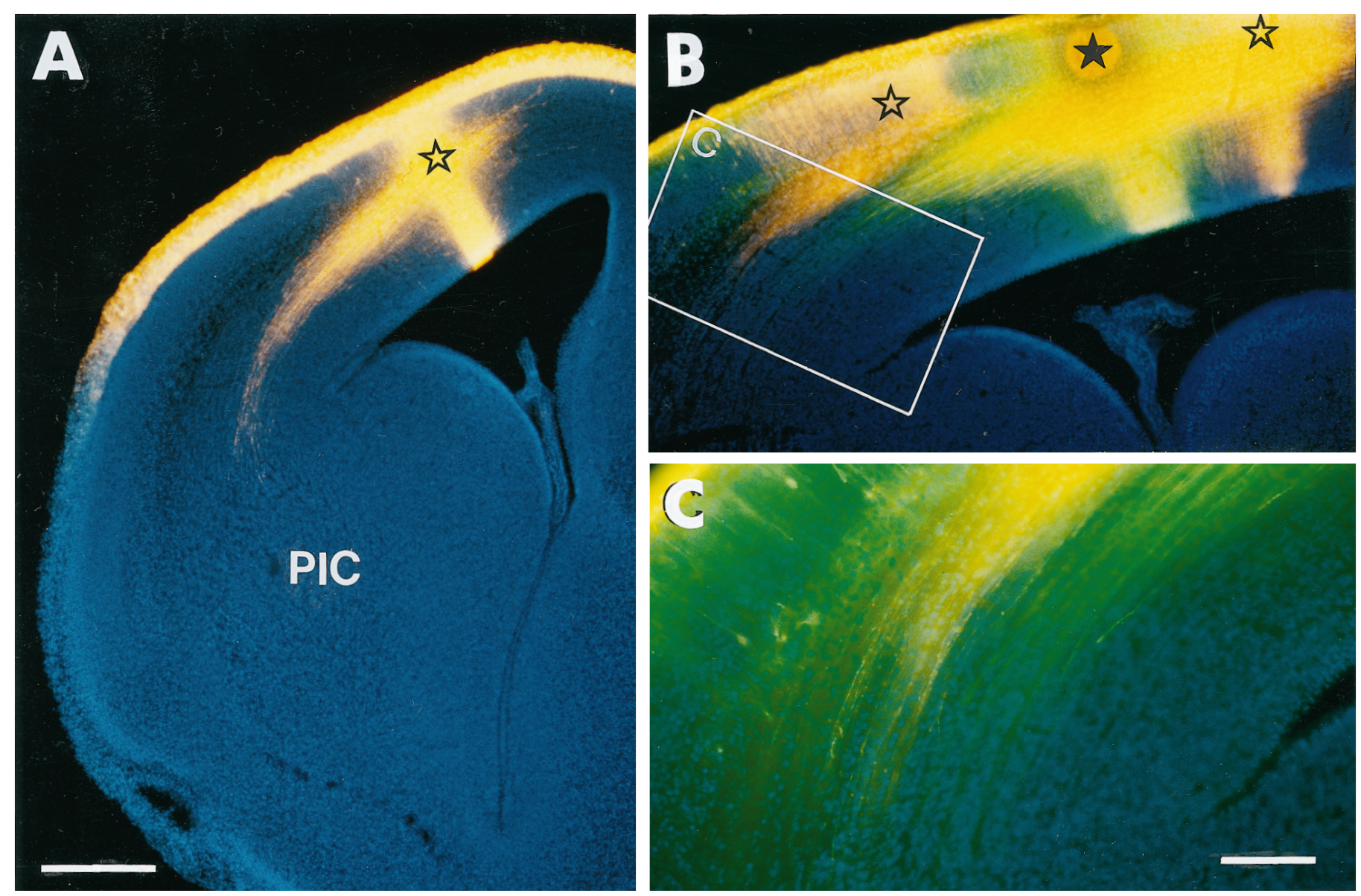

Figure 9. Carbocyanine crystal placements in the cortex at E15 revealed quite strict topographic order in the corticofugal projection within the intermediate zone and primitive internal capsule. A, Coronal, bisbenzimide-counterstained section from the left hemisphere of an E15 brain. A single DiI crystal (unfilled star) labeled a discrete bundle of early corticofugal axons descending through the middle of the intermediate zone toward the primitive internal capsule (PIC), as well as diffusely staining the cortical surface and labeling a column of radial processes, mainly radial glia. $B$, Three carbocyanine crystals, two DiI (unfilled stars), with a single DiO in between ( filled star in the middle), were placed along a coronal line in the cortical convexity of an E15 brain. Labeled fibers emanate from each crystal, descending through the intermediate zone, the differentially labeled fibers remaining separate and ordered along their path. Fibers labeled from the more lateral cortical crystal placement sites are more superficial in the intermediate zone and more advanced in their growth compared with those from dorsal sites, indicating a spatial and temporal ordering of outgrowth, in concordance with the ventral-to-dorsal maturation gradient across the cortex. $C$, Higher-power photomicrograph (taken from the region of the more ventral intermediate zone indicated by the white outline in $B$ ) showing mainly fibers from the most lateral of the three crystals. A few of the deepest axons are stained with $\mathrm{DiO}$ from the middle crystal. Note the strict ordering of axons, those from more dorsal sites lying deep to those from more lateral cortex. Scale bars: $A, B, 500 \mu \mathrm{m} ; C, 100 \mu \mathrm{m}$.

mature lateral segment of the cortex this population of firstgenerated cells is already being split into marginal zone and subplate by newly arrived migrating neurons of the emerging cortical plate (Fig. $7 E$ ). Interestingly, back-labeled cells are seen in both subplate and marginal zone of the lateral cortex at E14.5, indicating that some neurons from throughout the depth of the original preplate have axons in the primitive internal capsule at this age. Occasionally we observed a few back-labeled cells within the cortical plate itself, at least some of them with pyramidal morphology, but the vast majority are in the preplate or its derivatives (Fig. 7).

At E14.5, crystals placed in the internal capsule back-label cells only in the most mature, lateral neocortical segments. At later ages the territory of back-labeled cortical cells expands dorsally, indicating that axons from the preplate of more dorsal areas reach the internal capsule progressively later than those from more ventral regions.

Carbocyanine crystals implanted into the internal capsule after E16 back-label cells in the subplate but, interestingly, very few if any in the marginal zone, implying that the cells of layer 1 that originally projected to the internal capsule have either died or withdrawn their axons. At this stage cortical lamination is more advanced. Back-labeled pyramidal neurons are seen in both layers 5 and 6 , showing that corticofugal projection neurons in all these layers have now reached the internal capsule. Because cells in the cortex can be labeled before birth from the internal capsule but rarely if at all from the dorsal thalamus, we suspect that axons of subplate cells and even those from true corticothalamic neurons of layer 6 slow down or stop somewhere in the ventrolateral part of the diencephalon, medial to the primitive internal capsule, and do not enter the body of the dorsal thalamus prenatally.

\section{Anterograde labeling of the earliest corticofugal projections}

\section{E14-E15: establishment of the preplate "scaffold"}

The exact appearance of the cerebral wall (and the state of axon outgrowth, as revealed by crystal placements in the cortical surface) varies with position across the hemisphere, at any particular 
age, in line with the rostrolateral-to-caudomedial gradient of cortical development (Bayer and Altman, 1991). The rostrolateral cortex is $\sim 1.5 \mathrm{~d}$ ahead of the medial occipital cortex in its state of maturity until several days after birth. Unless otherwise stated, the following descriptions apply to crystal placements in the occipital cortex (putative area 17).

Crystal implantation in the surface of the occipital cortex early on E14 results in staining (to varying degrees) of the pial surface of the cortex and of a population of radial processes extending from the ventricular surface to the pial surface, with labeled bipolar, nucleated cell bodies in the lower half of the cerebral wall (Fig. $8 B$ ). The radial processes are presumably those of radial glia, and the somata probably include both progenitor cells of the ventricular zone and postmitotic, immature neurons, migrating toward the pial surface. In the most dorsal areas of cortex the preplate has apparently not yet begun to form; no cell bodies are present close to the pial surface. Nor are any outgrowing axons seen at this early stage.

During the 14th embryonic day, early-born cells begin to accumulate below the surface of the occipital cerebral wall to form the preplate. Crystal placement in the cortex at successively later times on E14 produces progressively more intense superficial staining, presumably concentrated in the membranes of these preplate cells. The first axons are seen growing out from these cells at mid-E14 (Fig. 8C), and they appear, virtually without exception, to be directed ventrally in the intermediate zone, heading toward the basal telencephalon and the primitive internal capsule, which they begin to enter during the second half of E14 (Fig. 8D). Wherever the crystal is placed, a substantial population of axons is labeled, and they form a fairly tight bundle of fibers running within and then directly beneath the subplate zone.

All the axons at the advancing front of the stained bundle end in expanded structures, which are indubitably growth cones rather than cell bodies, because chromatin staining reveals no nuclei within them. These growth cones exhibit complex filopodial morphology and are exceptionally large $(\sim 10 \mu \mathrm{m}$ across $)$ as they descend in the nascent intermediate zone (Fig. $8 F$ ). The bundle of axons originating at any particular point in the cerebral wall maintains its coherence quite tightly and follows a direct, smoothly curving path toward the primitive internal capsule. As they approach the perireticular cells below the lateral ganglionic eminence, the growing tips of the axons are somewhat more dispersed than the diameter of the bundle behind them, perhaps implying that they are entering territory with different properties.

Crystal placements in the occipital cortex on E14 never resulted in the back-labeling of thalamic cells, indicating that no thalamic fibers have reached the site of crystal placement at that age, in agreement with the results of anterograde labeling from the thalamus described above.

\section{Topography of the scaffold}

Examination of brains after multiple dye placements, in parasagittal or coronal rows across the cortical hemisphere, clearly demonstrated the temporal order of descending projections from the preplate, matching the gradient of maturation of cortical lamination (Bayer and Altman, 1991). Along each row, the more anterior and lateral the site, the more advanced the fiber outgrowth. These multiple injections also revealed the quite precise topographical organization of these early corticofugal projections; each individual crystal labeled a group of axons, forming a dis- crete, fairly tight bundle, and the spatial arrangement of the separate labeled bundles was maintained throughout their path.

Along the rostrocaudal axis, at these early stages, axons follow straight or slightly curved trajectories toward the primitive internal capsule and, hence, form a fan-shaped array converging on that region, maintaining topography correlated with the spatial separation of their sites of origin.

Topography is also preserved along the coronal axis (Fig. 9), despite the fact that all the fibers from any mediolateral strip of cortex share the same narrow portion of the intermediate zone. Figure $9, B$ and $C$, shows the separate, approximately parallel trajectories of the bundles of descending axons labeled by a coronal row of three crystals. At any point in the intermediate zone, the descending axons derived from more dorsal (latermaturing) parts of the hemisphere lie deep to those from more lateral (earlier-maturing) regions; the full array appears to extend through the entire thickness of the intermediate zone. Fiber order is, then, correlated with the time of initial outgrowth. This implies that, as new fibers grow down from progressively more dorsal regions, they take their place immediately adjacent and deep to the array of preexisting fibers, and that this order is preserved throughout the pathway. Thus the array of preplate axons approaching the ventral telencephalon forms a threedimensional representation of the entire surface of the hemisphere.

\section{Evidence that thalamic fibers grow over the scaffold of preplate axons within the internal capsule and intermediate zone}

\section{Combined double labeling of cortical and thalamic axons}

By late on E14, both advancing axon fronts, from occipital preplate and dorsolateral thalamus, reach a region in the basal telencephalon, at the lateral edge of the primitive internal capsule. The outgrowths of the two corresponding sets of axons, corticopetal and corticofugal, are remarkably synchronized and coordinated in their timing and spatial patterns.

Following the suggestion of McConnell et al. (1989) that the preplate pioneer fibers might play a part in subsequent axon guidance, we proposed that, after thalamic axons reach the internal capsule, they complete their navigation to the appropriate cortical region by growing over fibers that have descended from their target area-the "handshake" hypothesis (Blakemore and Molnár, 1990; Molnár and Blakemore, 1995). To examine this notion, we attempted to label corresponding regions of dorsal thalamus and occipital cortex with different dyes to see whether the two fiber systems do literally contact each other, intermingle, and appear topographicaly aligned within the intermediate zone. Results from such experiments performed at mid-E16 (Table 1) were rejected when we discovered that cell bodies in the thalamus were retrogradely labeled with the dye placed in the cortex. This was to be expected from the results of the thalamic tracing experiments described above, which showed that thalamic axons reach the occipital cortex at that age (Fig. 3). At even later stages, the picture becomes further complicated by the outgrowth of fibers from layers 6 and 5 of the true cortical plate (De Carlos and O'Leary, 1992; Miller et al., 1993). Our analysis with this doublelabeling technique was therefore restricted to the period from E14.5 to very early E16.

The fundamental problem with this approach is that the exact correspondence between developing thalamic and cortical loci cannot be established empirically until thalamic fibers have actually reached their target areas in the cortex, but after that point it 

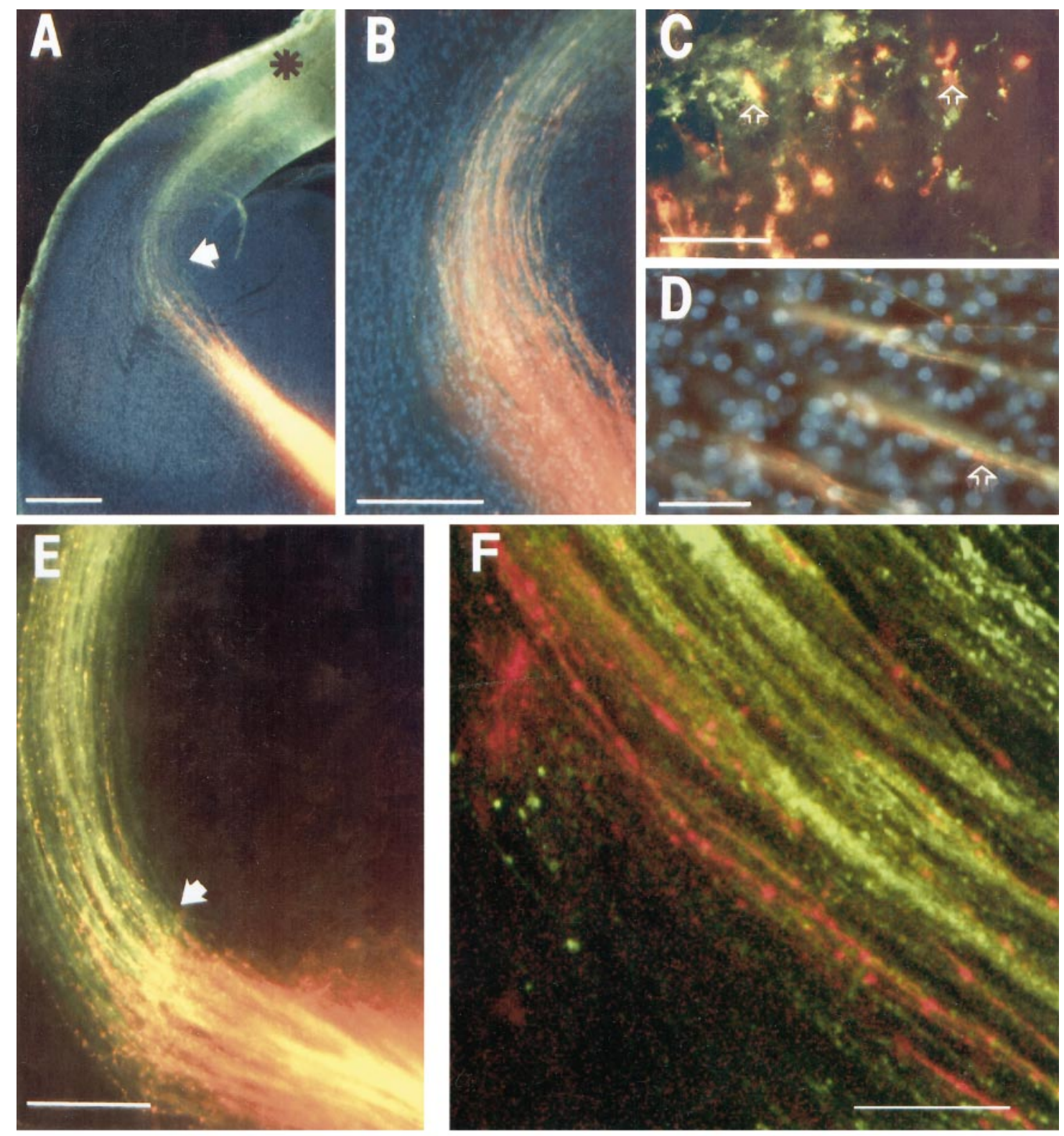

\section{G (stereo - pair)}
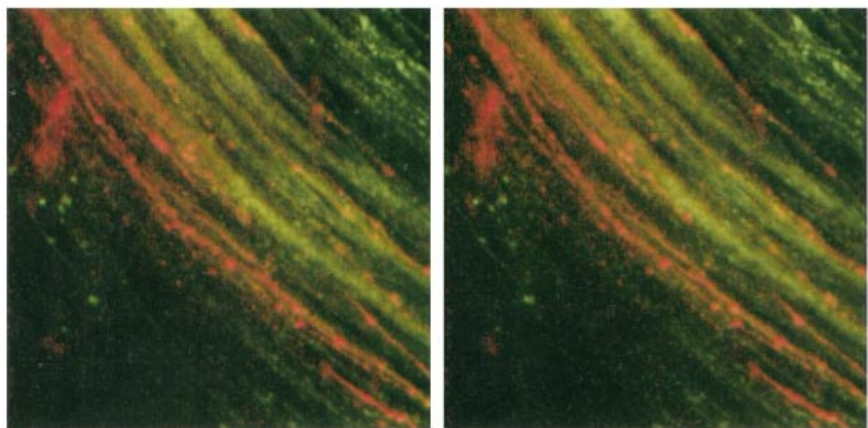

Figure 10. Evidence that thalamic and early corticofugal axons confront each other in the lateral internal capsule and share the same growth compartment within the intermediate zone (the handshake hypothesis; see Molnár and Blakemore, 1995). Shown are examples of the intermixing of differentially labeled early corticofugal and thalamocortical projections from four animals: at E15 $(C)$, E15.5 $(A, B, E, F)$, and very early E16 $(D)$. Crystals of two distinguishable carbocyanine dyes were placed, one into the dorsal thalamus and the other into the cerebral cortex of the same hemisphere, at or shortly after the time when cortical and thalamic efferents are converging at the lateral edge of the internal capsule. At these early stages the labeled thalamic fibers did not extend all the way to the cortex, and no back-labeled thalamic cells were seen in any of these specimens (Figure legend continues), 
is obviously very difficult reliably to label fibers arising from cells in that exact target region separately from the incoming thalamic axons. The handshake hypothesis demands that thalamic axons should intermingle only with axons from the corresponding region of the cortex; therefore, any misalignment of dye placements in the two structures would be expected to reveal spatially separate groups of labeled fibers. Indeed, in 11 of 26 (42\%) attempts to label matched cortical and thalamic sites with small crystal placements, from E14.5 to E15.5, the bundles of differently labeled descending and ascending axons lay close to each other within the intermediate zone but did not overlap extensively, just as in the results of Miller et al. (1993). However, because of the inherent difficulty in defining correspondence, negative results in such experiments are to be expected; even a small fraction of positive results would be definitive in demonstrating that the two axon systems grow through the same compartment within the intermediate zone.

In fact, in the majority of cases (5 of 8 at E14.5, 4 of 10 at E15, and 6 of 8 at E15.5) there was definite intermixing of a substantial fraction of the bundles of labeled preplate and thalamic axons in the ventral telencephalon and intermediate zone (Fig. 10 shows several representative positive examples). This was also true in one case at early E16 in which the labeled thalamic axons had not quite reached their target area, and no cells back-labeled by the cortical dye were seen in the thalamus. In several of these positive cases, the overlap of thalamic and corticofugal axons was extensive.

In these 16 successful double-labeling experiments, conventional fluorescence microscopy showed the two fiber arrays to be mixed together within individual $100-\mu \mathrm{m}$-thick sections, with differently colored fibers lying side-by-side in the same plane of focus. This was also the case in the thin optical sectioning afforded by confocal microscopy. For example, individual red and green fibers were seen close together in many of the 32 individual $1-\mu \mathrm{m}$-thick sections that were stacked to create the extended focus image of Figure $10 \mathrm{~F}$. The stereo pair derived from this data set (Fig. 10G) clearly shows corticofugal and thalamic fibers co-resident in single, bidirectional fascicles.

Cross-sectioning of the mixed bundles, in the intermediate zone lateral to the striatal anlage, confirmed the very close asso- ciation of the two populations, with individual descending and ascending axons often lying side-by-side, literally in contact. In Figure $10 C$, for instance, there is a considerable area of overlap, with some red (thalamic) and green (corticofugal) axons so close together that they appear optically fused as yellow.

\section{Labeling of cortical neurons and thalamic axons as they approach the subplate}

Between E15 and E16, a dye crystal in the primitive internal capsule labels not only early corticofugal axons and their cell bodies (retrogradely) but also thalamic axons growing toward the cortex (anterogradely). In 42 such cases we examined the relationship between ascending and descending axons that pass through the same region of the internal capsule, especially in brains that were cut in a plane (near-coronal) chosen to be parallel to the axon arrays close to the labeled region of cortex.

Figure 11 shows results from an E15.5 brain in which a crystal of DiI had been placed in the medial part of the primitive internal capsule. At the rostrocaudal level of the crystal site, an ordered array of intensely stained, thick thalamic axons, identified by the growth cones at their tips, is advancing into the basal part of the intermediate zone. A number of thinner axons, intermingled with this mass of thalamic fibers, extend all the way back to the cortex, some of them unequivocally identified as corticofugal by being followed back, in serial confocal sections, to labeled cell bodies in the subplate (Fig. 11C). Further rostral, where the entire pattern of growth is more advanced, a dense array of thin axons without growth cones leads back to a population of back-labeled cells in the cortical subplate and some in the lowest part of the cortical plate. Many of these fine, labeled axons can be traced back individually to their cells of origin. Among this ordered descending array of corticofugal axons, thicker axons, presumed to be thalamic, because many of them end in definite growth cones within the section, are seen growing up toward the cortex, running parallel to and in intimate association with corticofugal fibers (Fig. 11D). There is no tendency for the two types of axons to separate from each other, even as the thalamic axons enter the subplate layer.

Figure 12 is an enlarged view of the region of the confocal

\section{$\leftarrow$}

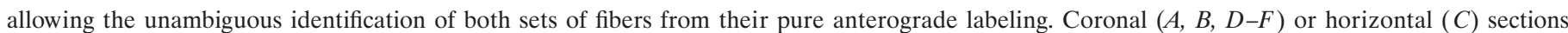

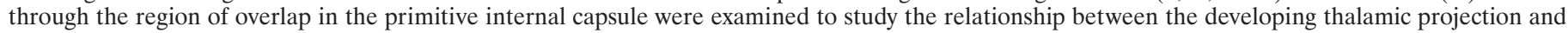

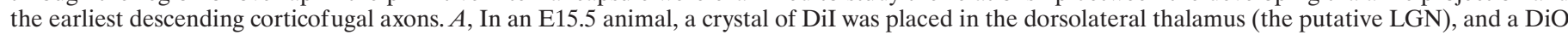

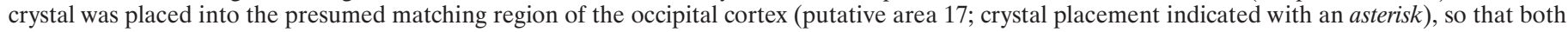

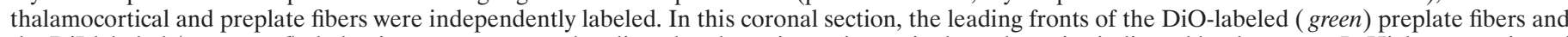

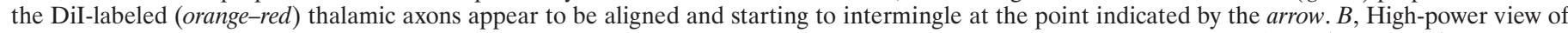

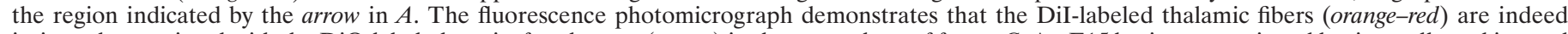

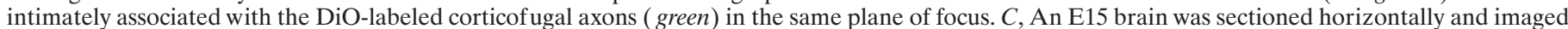

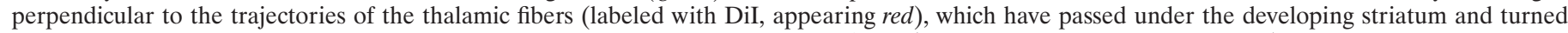

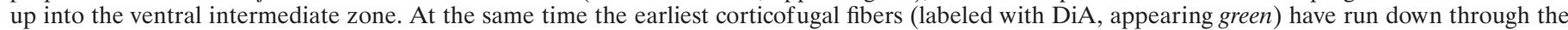

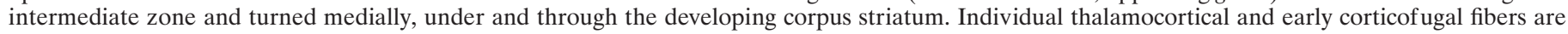

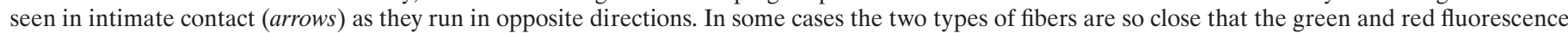

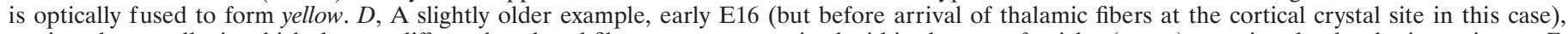

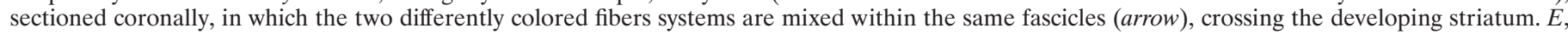

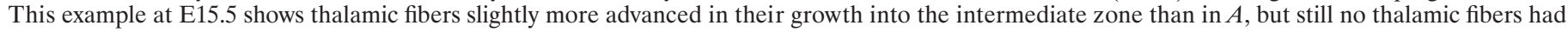

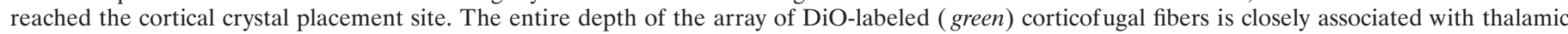

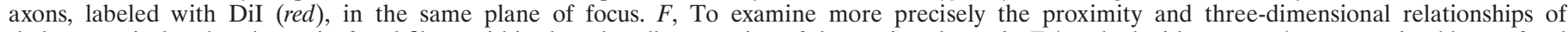

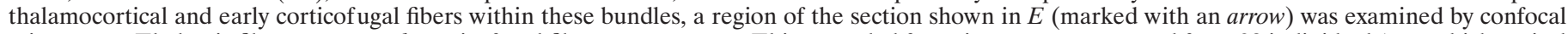

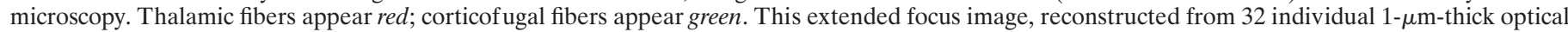

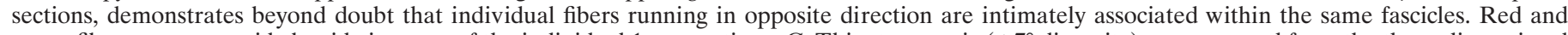

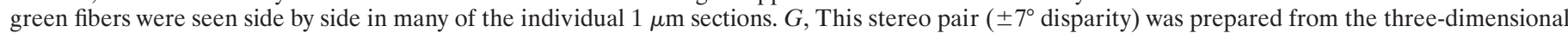

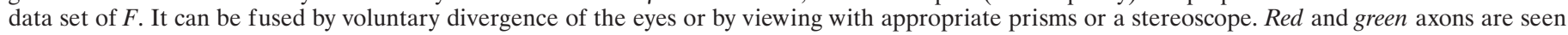
closely approximated throughout the depth of the section. Scale bars: $A, 250 \mu \mathrm{m} ; B, E, 100 \mu \mathrm{m} ; C, D, 50 \mu \mathrm{m} ; F, 10 \mu \mathrm{m}$. 

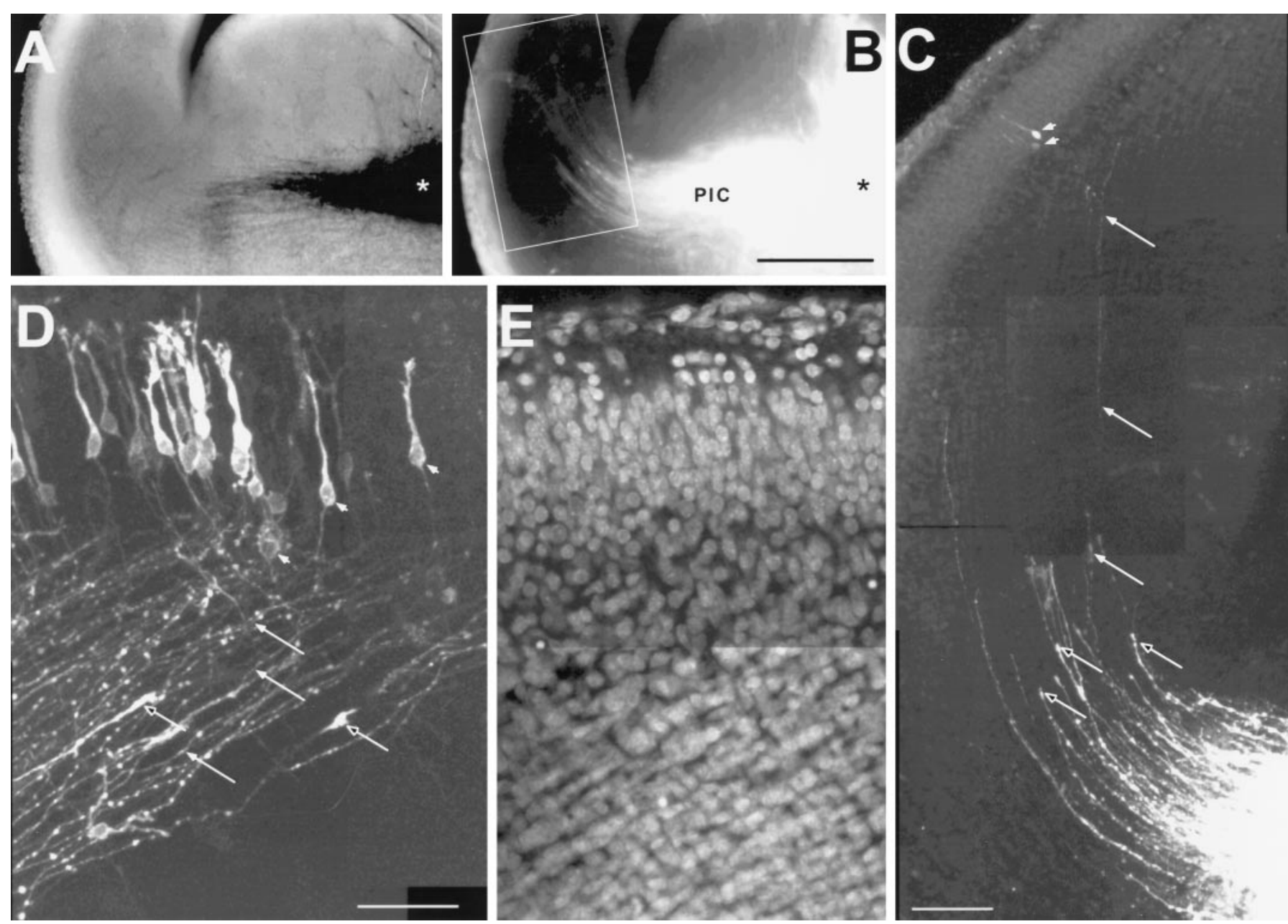

Figure 11. To examine the relationship between early corticofugal axons and thalamocortical afferents as the latter approach the target region of the cortex, a single DiI crystal was placed in the internal capsule of an E15.5 brain. $200-\mu \mathrm{m}$-thick sections were cut in a vertical plane, angled $45^{\circ}$ forward from the coronal plane, approximately parallel to the axon pathway between the internal capsule and the cortex in the middle of the hemisphere. Confocal microscopic reconstructions from these specimens reveal that thalamic fibers (labeled anterogradely) and corresponding early corticofugal efferents (labeled retrogradely from the same dye crystal) occupy the same region of the intermediate zone, and that the thalamic fibers grow among the corticof ugal axons right up to the target region. $A$, Low-power view of a section through the crystal placement site (asterisk) stained with acridine orange. $B$, Fluorescent micrograph of the same view as in $A$ reveals the mass of DiI-labeled fibers extending from the primitive internal capsule (PIC) into the intermediate zone and up toward the lateral wall of the telencephalon. $C$, Higher-power confocal microscopic reconstruction of the outlined area in $B$ from the intermediate zone under the lateral sector of the cortex. This extended focus projection reveals anterogradely labeled thalamocortical fibers as well as retrogradely labeled corticof ugal axons and their cell bodies. The thalamocortical fibers, many of which were unequivocally identified by following them to growth cones at their tips (examples are marked with unfilled arrows), form a broad array as they enter the intermediate zone; note that they appear relatively thick and intensely stained. Mixed with these thalamic axons in the basal telencephalon is another array of much finer axons, hardly visible at this magnification, extending all the way up to the cortical subplate ( filled arrows), many of which were definitively identifiable as corticofugal because they could be traced back to labeled cell bodies in the cortical subplate or occasionally in the very lowest part of the cortical plate ( filled arrowheads). D, Higher-resolution confocal reconstruction from directly below the lateral cortex in a slightly more anterior section, where thalamic axons have advanced further toward the cortical subplate. A large number of neurons ( filled arrowheads) are back-labeled in the subplate and in the lowest part of the cortical plate itself, some even with pyramidal morphology. The most advanced of the thalamic fibers are approaching this region, ending in large growth cones (open arrows), just $100 \mu \mathrm{m}$ below the cortex. These thalamic fibers are running parallel to and in close association with finer, retrogradely labeled early corticof ugal projections ( filled arrows), many of which can be traced back to their labeled somata. This intimate mixture of thalamocortical and corticofugal axons runs as a broad swathe of parallel fibers through the intermediate zone and into the subplate, with no obvious segregation into two separate compartments. $E$, Acridine orange counterstaining of $D$ demonstrates cell layering in the cerebral wall. Scale bars: $A$, $B$, $500 \mu \mathrm{m} ; C, 100 \mu \mathrm{m} ; D, E, 50 \mu \mathrm{m}$.

image of Figure $11 \mathrm{D}$, in which the outlines of several identified corticofugal axons have been filled in black, together with their cells of origin, whereas the outlines of some of the definite thalamic fibers, identified by their growth cones, are shaded white. The two types of identified axons run parallel, in close association, filling the same compartment of the intermediate zone, as the thalamic fibers approach within $100 \mu \mathrm{m}$ of the cortex.

\section{Shared topography of early corticofugal and thalamocortical projections}

Although exactly corresponding thalamic and preplate axons cannot reliably be separately labeled after thalamic fibers have reached the subplate layer, further support for the handshake hypothesis can be drawn from experiments involving crystal placement in the cortex alone at slightly later ages. Fibers from the putative LGN reach the subplate of the occipital pole of the 


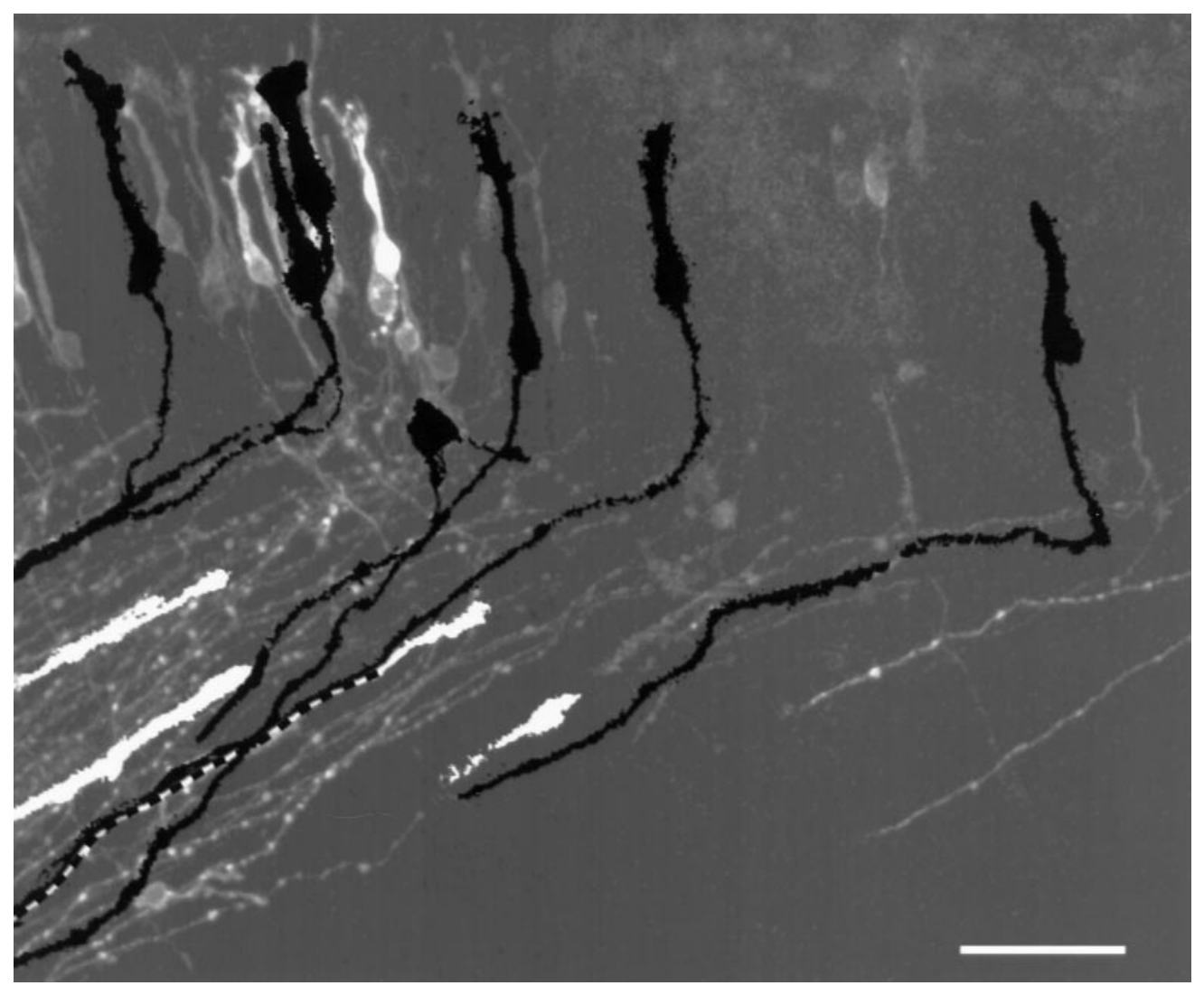

Figure 12. The trajectories of individual thalamic and cortical axons in the region shown in Figure $10 D$ were separately traced within the threedimensional data set. Two masks were constructed by marking the suprathreshold pixels of seven corticofugal axons, traced to somata in the subplate or lower cortical plate (black), and of five thalamic axons, with identified terminal growth cones (white). These profiles were finally projected and merged with the original data set. In one case, a cortical axon and a thalamic axon, identified by their emergence from a cell body and terminal growth cone, respectively, were so closely juxtaposed that they could not be separately resolved (interrupted line). Scale bar, $50 \mu \mathrm{m}$. cortex at E16 (Fig. $3 G$ is representative for all experiments; $n=$ 8). In accordance with this, a single dye crystal in the occipital cortex at E16 leads to the labeling not only of fibers, extending through the telencephalon and diencephalon, linking the two structures (Fig. 11C), but also of cell bodies in the LGN itself (especially clear after relatively long incubation periods). Therefore the fibers labeled from any such single site in the cortex must be a mixed population of anterogradely labeled, corticofugal axons and retrogradely labeled, thalamocortical fibers. And, by definition, they must be spatially corresponding. If these two sets of axons were to take very different routes through the telencephalon, any single crystal should stain two or more distinctly separate bundles.

We therefore examined the axon arrays labeled by small dye placements at various points on the cortex, at ages from E16 onward, following the axons in serial coronal or horizontal sections with conventional fluorescence and confocal microscopy. As well as viewing these serial sections, we made three-dimensional reconstructions from thin confocal optical sections at various magnifications to reveal more of the fine and gross topography.

Unfortunately, even very small crystal placements lead to a wide halo of label around the uptake site itself, especially after the relatively long incubation periods that we used to obtain good retrograde labeling of thalamic cell bodies. This halo consists partly of intrinsic cell and fiber staining in the cortex itself, partly of direct labeling of radial processes and cells in the subadjacent intermediate and subventricular and ventricular zones, and partly of background dye that has presumably diffused radially around the crystal. The fact that the total bundle of fibers emerging from such a halo and running down toward the internal capsule is always much smaller than the diameter of the halo implies that the effective uptake site for fiber labeling is very much smaller than the halo, as with most axonal tracers.

Although the labeled fibers could not be traced reliably within the dye halo itself, very close to the uptake site, they could be clearly resolved over the remainder of the pathway, throughout most of the intermediate zone, the internal capsule, and right into the thalamus. The mixed descending and ascending axons labeled by any small crystal placement in the cortex at E16 form a discrete bundle rather than two separate fiber tracts. This single, tight bundle is maintained throughout its trajectory within the intermediate zone and primitive internal capsule, over the region containing both sets of axons. The stained bundle narrows as it approaches and runs through the internal capsule, where, for a small crystal site, it can be as tiny as $50 \mu \mathrm{m}$ in diameter (Fig. 13B). A fine ribbon of stained fibers extends into the diencephalon and follows a smoothly curving trajectory to the appropriate region of the thalamus, ending in a cluster of back-labeled cells.

From serial confocal microscopic optical sections, we made high-resolution reconstructions to reveal the fine topography of stained fibers within the entire bundle between thalamus and cortex. This technique did not quite allow us to follow individual axons all the way from the implantation site to the thalamus, but it did show that the bundle consists of an approximately parallel set of axons throughout its course, with very little crossing over within the array (just as in the images of separately labeled thalamic and preplate axons seen at earlier stages; Fig. 10F).

Because this method does not allow discrimination of the two classes of fibers, it is impossible to be sure whether the segment of the labeled bundle within the diencephalon contains corticof ugal axons. However, the bundle usually appears thinner and yet less densely packed within the diencephalon (Fig. 13C), suggesting 

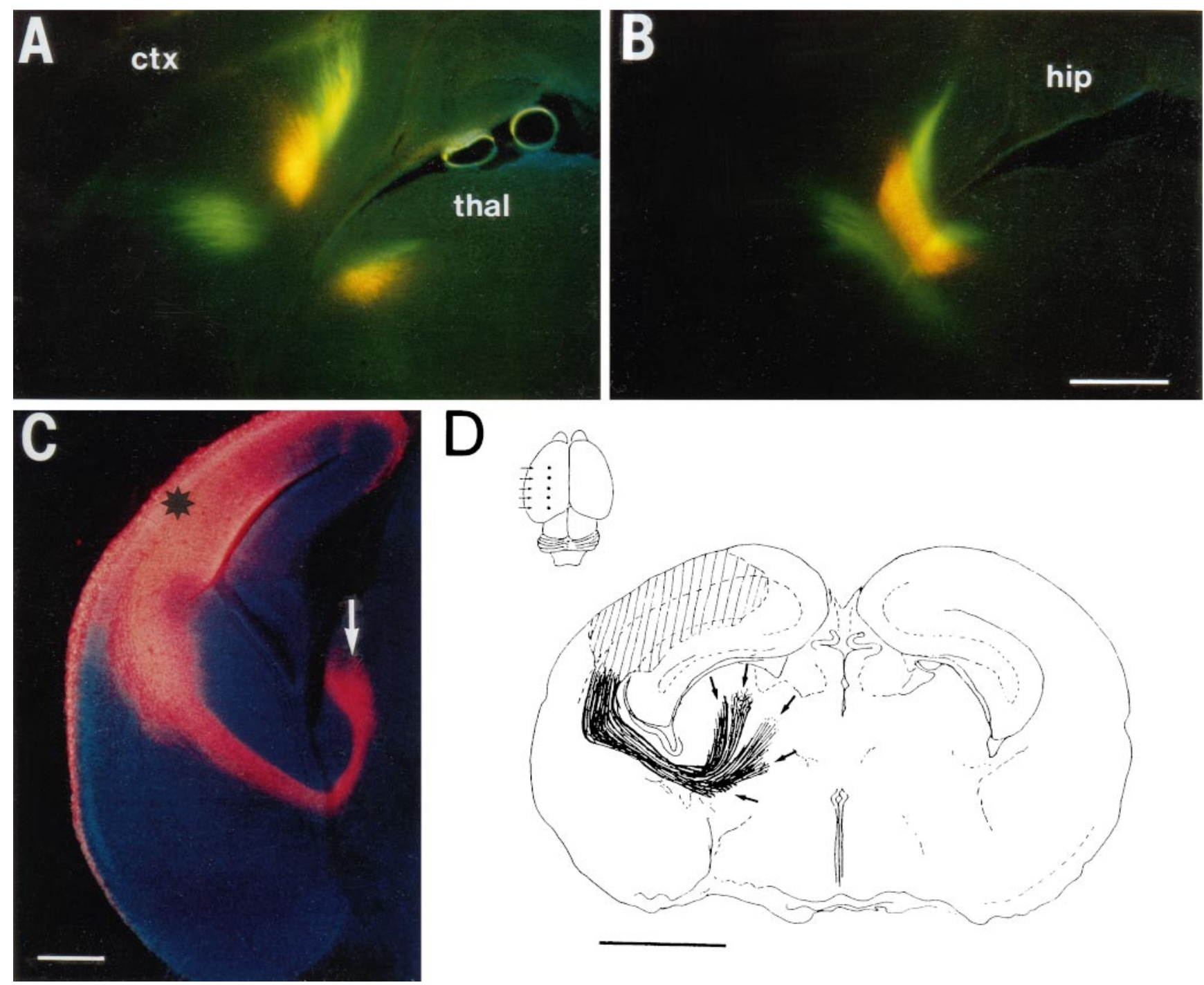

Figure 13. Further evidence for common topography and close association of early corticof ugal and thalamocortical projections comes from combined anterograde and retrograde tracing from the cortex at and after E16. $A, B$, Small crystals of three different dyes (DiA, DiI, and DiAsp) were placed at points in a parasagittal row along the right hemisphere at E20, and the three distinct bundles of axons (containing both corticof ugal and thalamocortical axons) were followed in $100-\mu \mathrm{m}$-thick horizontal sections. (Rostral is to the left, lateral is $u p$ ). There was no evidence for substantial mixing or crossing of these bundles at any point in the pathway between cortex $(c t x)$ and thalamus (thal). $A$ is slightly superior to $B$ and shows the three bundles within the ventral telencephalon (top), converging toward the internal capsule and diverging again within the diencephalon (bottom), heading toward different points in the thalamus. $B$, at the level of the hippocampus (hip), shows the three distinct bundles funneling through the narrow junction between telencephalon and diencephalon. $C$, A single crystal of DiI placed in the left occipital cortex of an E16 brain produced a large halo of diffuse stain around the implantation site (asterisk) but nevertheless labeled a single, quite narrow fiber bundle. This double exposure shows both DiI labeling (red) and bisbenzimide counterstaining. The bundle of fibers runs in the intermediate zone, turns medially, narrowing as it passes through the internal capsule, and then turns quite sharply upward as it enters the diencephalon, sweeping up to the dorsolateral part of the thalamus. In higher power, a small group of back-labeled cells is seen in the LGN (arrow). It is likely that the segment of the bundle within the thalamus itself consists only of thalamocortical axons (see tracing from the internal capsule in Results). $D$. Five separate crystals of carbocyanine dye were placed in a parasagittal row along the left hemisphere of an E20 animal (indicated by arrows in the inset diagram of a dorsal view). Bundles of axons linking cortex and thalamus (containing both corticof ugal and thalamocortical axons) were revealed in coarse, $250-\mu \mathrm{m}$-thick coronal sections. Each ran to a different region of the thalamus, ending in a small group of back-labeled cells. At the level of the section drawn here, the five distinct bundles were all clearly visible, passing through the primitive internal capsule without obvious mixing or crossing; the tip of each bundle is marked with an arrow. Back-labeled cells associated with the two bundles from the most caudal crystal placements lay in this plane; the bundles labeled by the more anterior crystals terminated more ventrally and medially in the thalamus. Scale bars: $A, B, 500 \mu \mathrm{m} ; C, 250 \mu \mathrm{m} ; D, 1 \mathrm{~mm}$.

that it contains fewer fibers. And the fact that we never succeeded in back-labeling subplate cell bodies with small dye placements restricted to the dorsal diencephalon (see above) also implies that their axons do not reach the relay nuclei of the thalamus itself. Therefore, the fine bundle of stained axons running within the thalamus, toward the group of back-labeled thalamic neurons, probably consists entirely of the retrogradely stained axons of those cells.

It is important to note that the topographic arrangement of the fiber bundles in the diencephalon and the cell clusters in the thalamus itself, after cortical placement at E16, is entirely compatible with the picture derived from the implantation of crystals 

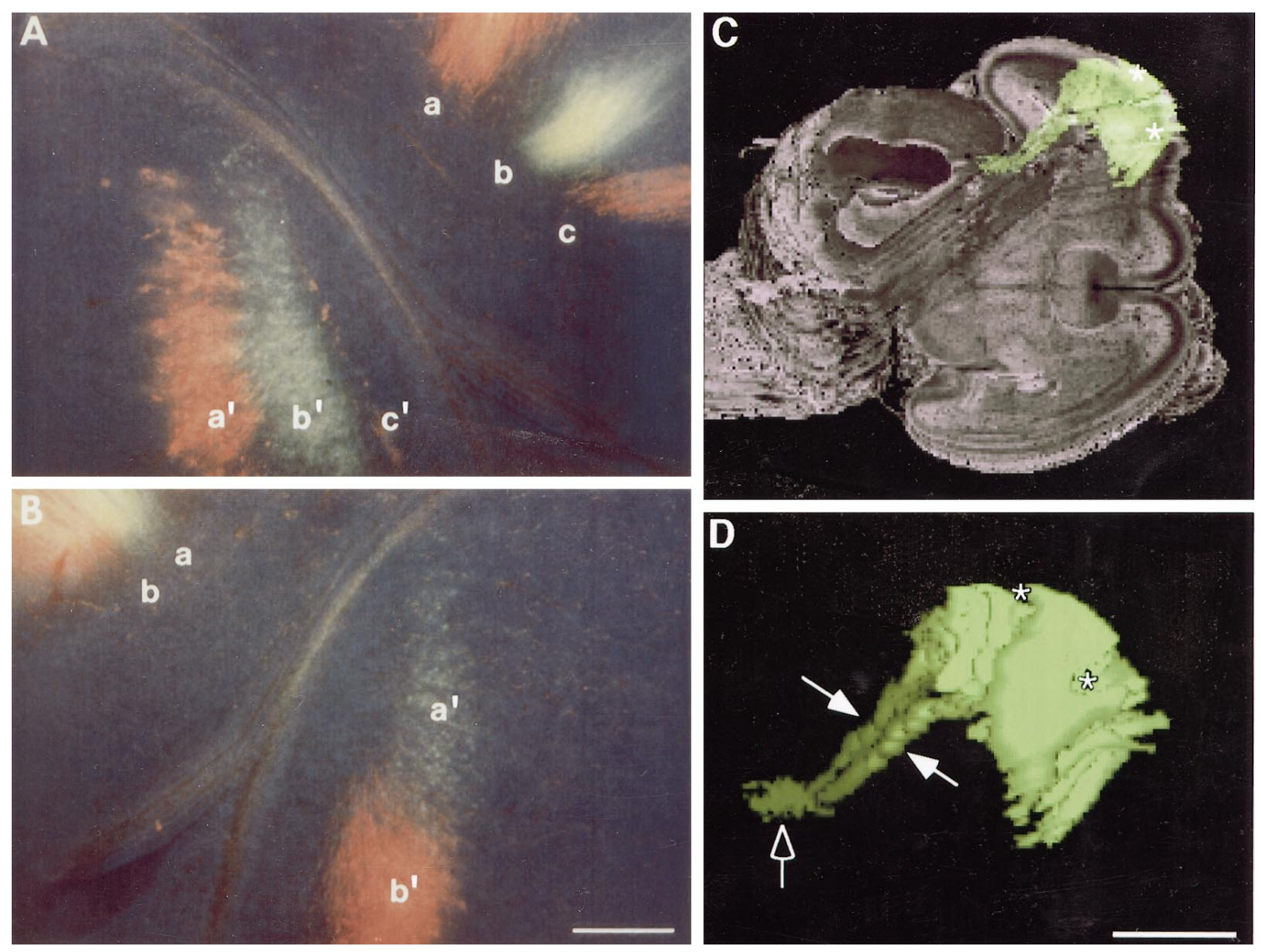

Figure 14. A, B, Bundles of closely mixed corticof ugal and thalamocortical axons were revealed by applying three crystals of carbocyanine dye (DiI, DiA, DiI) in a parasagittal row along the right hemisphere and two crystals along a coronal line in the left hemisphere (DiA ventral to DiI) of the same E16 brain. $A, B$, Multiple-exposure fluorescence photomicrographs of the left $(A)$ and right $(B)$ hemispheres taken from a single $100 \mu \mathrm{m}$ horizontal section just superior to the junction of telencephalon and diencephalon. $A$, Right hemisphere (top, rostral; left, medial). The three distinct bundles ( $a-c$, from rostral to caudal) are clearly visible within the ventral telencephalon (top right). The corresponding groups of back-labeled thalamic cells, visible on the left, form anteroposterior slabs $\left(a^{\prime}-c^{\prime}\right)$. Note the $90^{\circ}$ rotation in topography between telencephalon and thalamus, which occurs in the ventral diencephalon; the anterior-to-posterior sequence of cortical loci $(a-c)$ corresponds to a mediolateral sequence of thalamic slabs $\left(a^{\prime}-c^{\prime}\right)$. $B$, Left hemisphere (top, rostral; right, medial). In the ventral telencephalon (top left) the two fiber bundles appear partly superimposed, although they are separate in the orthogonal plane; the bundle from the more ventral crystal ( $a$, DiA; yellow-green $)$ lies deep to that from the more dorsal crystal $(b$, DiI; orange). The two corresponding back-labeled cell groups $\left(a^{\prime}, b^{\prime}\right)$, although clearly segregated, line up along a continuous anteroposterior slab in the thalamus (bottom right). The ventrodorsal sequence along the coronal line on the cortical convexity corresponds to an anterior-to-posterior sequence of thalamic cells along one continuous slab. Scale bar: $A, B, 300 \mu \mathrm{m}$. $C, D$, Three-dimensional reconstructions of the entire pathway between thalamus and cortex at E16 to show the shared topology of early corticofugal and thalamic projections. Frontal cortex was selected because the pathway is relatively straight. Two crystals of DiI were placed on a coronal line near the frontal pole of the left hemisphere, labeling both thalamic and preplate axons (probably with very little involvement of fibers from cells of the true cortical plate at this early age). After 6 weeks incubation, to allow complete anterograde and retrograde diffusion, $75-\mu \mathrm{m}$-thick horizontal sections were cut and counterstained with acridine orange. Each section was imaged in a fluorescent microscope with both rhodamine and fluorescein filters, and a series of 43 images was digitized, aligned, and superimposed for the reconstruction of a three-dimensional data set. $C$, The reconstructed stack of superimposed sections has been rotated around its sagittal axis to tilt the left hemisphere up slightly; the tilted brain is viewed from above (rostral to the right). To expose all the DiI labeling, which appears green, surrounding parts of the brain have been "dissected" away in the reconstruction, with the central part "scooped out," right down to the internal capsule. The two labeled axon bundles are clearly visible, running backward, medially and down in the intermediate zone, through the internal capsule and into the anterior thalamus. $D$, In this enlarged view of the DiI labeling alone, the two fiber bundles are seen to be clearly discrete, running parallel to each other, even through the constriction of the internal capsule (between the filled arrows). The back-labeled cells in the thalamus form a continuous slab (unfilled arrow), oriented anteroposteriorly. Scale bar (on $D$ ): $C, 2 \mathrm{~mm} ; D, 1 \mathrm{~mm}$.

in the thalamus $\sim 1 \mathrm{~d}$ earlier, shortly after axon outgrowth (compare Figs. 2, 13D). This strengthens the conclusion that outgrowing thalamic axons form a highly ordered array, even within the diencephalon itself.

Taken together with the double-labeling experiments and those in which both descending and ascending axons were labeled from the internal capsule, these results suggest that (1) the topography of the scaffold of early corticof ugal axons within the intermediate zone and basal telencephalon is extremely similar in form to that of the thalamocortical fibers; (2) each set of fibers appears to be distributed, in order, within the entire depth of the intermediate zone; and (3) ascending and descending axons share the same compartment and are intermingled, through an extensive region of the ventral telencephalon, to within a very short distance of the target area for the thalamic fibers.

It might be argued that some mixing of the two sets of axons is inevitable if they share a single extracellular growth compartment, especially as they approach the constriction of the internal capsule. However, compelling evidence for global, topographically ordered intermeshing of the two axon arrays comes from the 

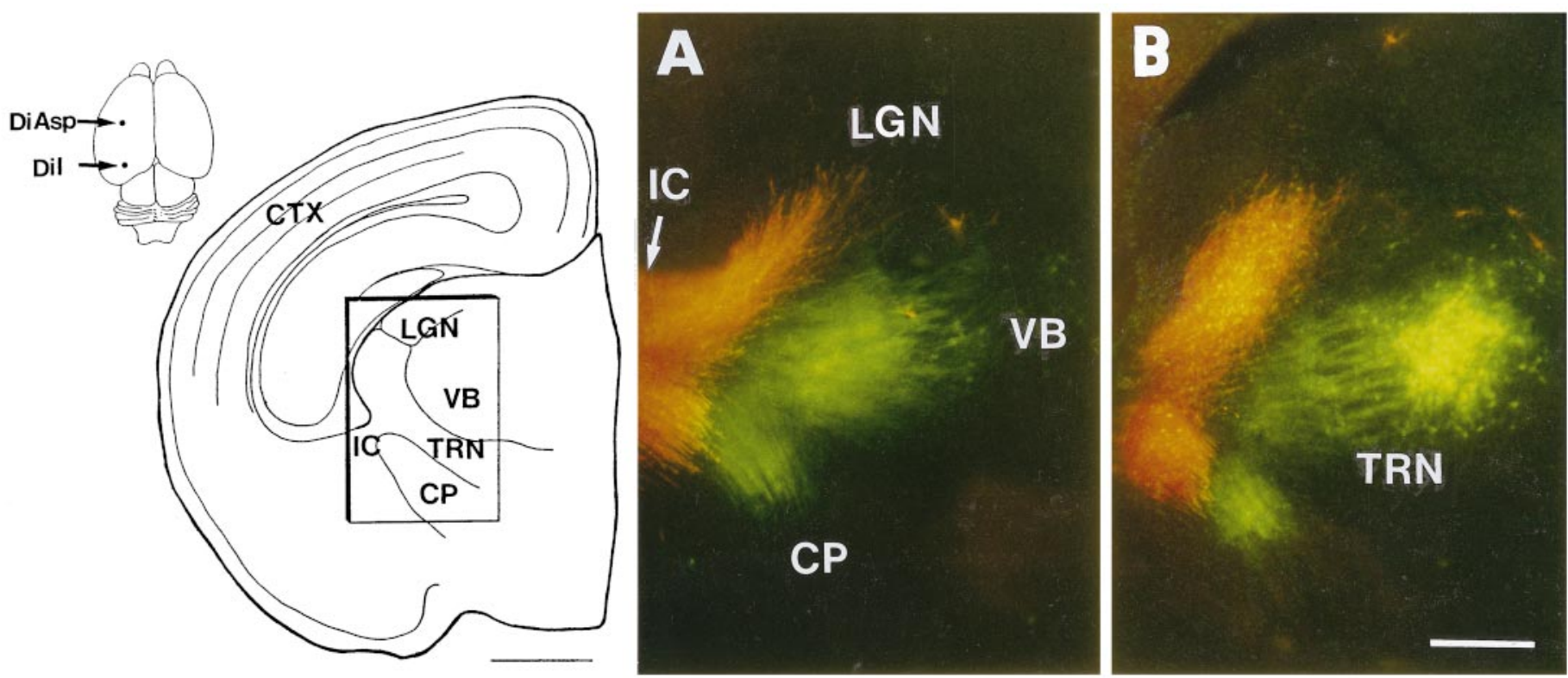

Figure 15. Divergence of corticof ugal projections at later ages. Two different carbocyanine crystals were placed $3 \mathrm{~mm}$ apart along a parasagittal axis in the convexity of the left hemisphere of a P2 rat (DiI in the putative visual cortex, DiAsp in the presumed somatosensory cortex), as shown in the inset diagram. $A, B$, Double-exposure fluorescence micrographs of two adjacent $100-\mu \mathrm{m}$-thick coronal sections $(A$ rostral to $B)$ from the region of the ventral diencephalon, just medial to the internal capsule (IC), indicated by the box in the camera lucida drawing on the left. Through the telencephalic portion of the pathway, each dye labels a discrete, coherent bundle of fibers, and the two bundles do not mix as they sweep through the internal capsule, implying that all the projections labeled at this late stage (corticofugal, from layers 6 and 5, as well as preplate and thalamocortical) share the same overall topography. But on the diencephalic side of the internal capsule, each labeled bundle divides into two: a component that continues into the thalamus (thalamocortical and corticothalamic fibers) and a ventral branch (presumably containing the axons of the corticotectal, corticopontine, and corticospinal projections), which descends into the cerebral peduncle. This divergence is visible as early as E18-E19. A, Axons labeled with DiI (orange) turn up toward the LGN, whereas those labeled with DiAsp ( green) run medially toward the ventrobasal thalamus $(V B)$. Each bundle has a ventral branch that diverges into the cerebral peduncle $(C P)$. Note that the differently colored descending branches remain segregated from each other, suggesting that fiber order is maintained in this portion of the brainstem projection. $B$, Just posterior, each thalamic component is approaching the group of back-labeled cells in its own nucleus. As this level the thalamic reticular nucleus $(T R N)$ lies directly below the ventrobasal thalamus. CTX, Cortex. Scale bars: camera lucida drawing, $1 \mathrm{~mm} ; A, B, 300 \mu \mathrm{m}$.

experiments involving multiple cortical dye placements (Figs. $13 A, B, D, 14)$, in which the individual bundle of fibers labeled by each crystal can be followed all the way to the thalamus. At E16, each distinct bundle is unitary and clearly separate from the others, even as they converge and pass through the primitive internal capsule. This is most clearly shown in Figure 14, $C$ and $D$, which is a three-dimensional reconstruction of the entire trajectory of two closely spaced fiber bundles running between frontal cortex and anterior thalamus.

Within the resolution of this method, the apparently single bundle labeled from any one crystal could conceivably represent closely adjacent but nonoverlapping strands of thalamocortical and corticofugal axons. But obviously, close proximity of corresponding parts of the two axon arrays without overlap of any part is incompatible with the overall topography and closely packed fiber ordering seen within each array. Ascending thalamic axons appear, then, to follow the same topographically ordered paths through the telencephalon as do their counterparts descending from the subplate. Either the two arrays are systematically very slightly misaligned from each other, or, as demanded by the handshake hypothesis, they are spatially corresponding.

Retrograde labeling of thalamic neurons: global topography of the projection

Although it is impossible to resolve the individual components of the mixed fiber population labeled from a cortical implantation site, important inferences about their interrelationships and topography can be drawn from the patterns of retrograde labeling of thalamic cell bodies. In horizontal sections, the thalamic cells back-labeled from any single crystal in the cortex tend to form an anteroposteriorly elongated slab. Different crystals placed in a parasagittal line along the hemisphere at E16 revealed that more anterior cortical regions are innervated by more medial slabs within the developing thalamus, whereas more posterior cortical regions receive from more lateral thalamic slabs (Fig. 14A). In concordance with this, any two adjacent cortical implantation sites arranged along a coronal line produced a pair of elongated groups of back-labeled thalamic cells aligned to form a more-orless continuous anteroposterior slab, the rostral portion of which projects more ventrally in the cortex (Fig. 14B).

\section{Other corticofugal fiber systems}

De Carlos and O'Leary (1992) showed that pyramidal neurons of the lowest part of the true cortical plate (presumably layer 6) start to extend axons into the white matter as early as E15 in the rat. Interestingly, at E16, axon bundles labeled from the cortex were always unitary throughout their visible course, although the telencephalic portion should have contained at least some axons of layer 6 cells as well as those of preplate cells and thalamocortical fibers. This surely means that all these axon systems run close to each other. And the separate, ordered arrangement of bundles resulting from multiple dye placements implies not only that all the component axon types share the same growth compartment but also that they are distributed according to the same overall topography. 
For the portion of the pathway running through the intermediate zone, this general pattern persists even after E18-E19, when there is also significant extension of axons from layer 5 of the cortical plate, ultimately directed toward structures below the diencephalon (De Carlos and O'Leary, 1992; Mitrofanis and Guillery, 1993). Again this implies topographically ordered mixing of all the fiber systems within the telencephalon. However, the capacity of the single-crystal technique to demonstrate divergence of axon populations when they do exist is established by the fact that, at these later ages, each single dye placement in the cortex does indeed reveal a fiber bundle (presumably consisting of layer 5 cell axons) that diverges from the main labeled tract into the posterior extension of the primitive internal capsule to form the putative cerebral peduncle (Fig. 15). At this stage, dye placed in the posterior part of the primitive internal capsule exclusively labels cell bodies in layer 5 of the cortical plate itself, presumably the cells of origin of corticotectal, corticopontine, and corticospinal projections. Thus, it seems that these nonpioneering, laterdescending fibers from layer 5 share the same gross topography as the earlier ascending and descending projections within the telencephalon (although possibly systematically slightly displaced from them), but that the layer 5 axons follow radically different trajectories in the ventral telencephalon, presumably responding to guidance cues in that region.

\section{DISCUSSION}

\section{Topographic order of thalamic axons}

The arrangement of the bundles of thalamic axons labeled by crystal placements in the thalamus, shortly after their outgrowth (Fig. 2), or in the cortex, immediately after their arrival (Figs. $13 D, 14 C, D)$, suggests that they maintain ordered topography, running as an approximately parallel array along their entire route, with a single $90^{\circ}$ rotation of the array as it leaves the ventral diencephalon (Fig. 2). Fibers from parasagittal slabs of thalamic cells innervate loci lying along approximately coronal lines across the cortex; more ventral cortex is innervated by cells lying more rostral in the thalamic slab, and the more anterior the coronal strip, the more medial (and slightly ventral) is the corresponding thalamic slab (Fig. 14). The global topographic relationship between thalamus and cortex appears very simple at this stage, before the clear segregation of thalamic nuclei. The entire volume of the thalamus maps continuously onto the cortical sheet.

The initial pattern of thalamocortical innervation could subsequently be altered through differential growth in thalamus or cortex, irregular ingrowth of thalamic fibers, selective growth and retraction of their side branches (Naegele et al., 1988; Ghosh and Shatz, 1992), or activity-dependent redistribution of thalamic afferents in the cortex (see Shatz, 1990; Molnár, 1998).

\section{Initial guidance of thalamic outgrowth}

The ordered outgrowth from the thalamus might depend simply on intrinsic interactions between neighboring thalamic fibers. However, a number of other neural systems in this region could be players in the process of guidance. Like Mitrofanis and Baker (1993), we saw cells of the thalamic reticular nucleus backlabeled from the dorsal thalamus at E14 and also small numbers of back-labeled neurons within the primitive internal capsule, presumably belonging to the "perireticular" cell group of Mitrofanis (1992) but projecting into the thalamus earlier than previously described (Mitrofanis and Baker, 1993; Mitrofanis and Guillery, 1993). Thus, at least two populations of fibers have reached the thalamus when thalamic axons are emerging, and they could conceivably play a part in guiding that initial outgrowth.

\section{The "handshake" hypothesis: possible guidance by preplate axons}

Axons from preplate cells pioneer a pathway through the intermediate zone (McConnell et al., 1989; Blakemore and Molnár, 1990; De Carlos and O'Leary, 1992; Erzurumlu and Jhaveri, 1992; Miller et al., 1993; Molnár et al., 1998). Rows of dye crystals placed in the cortex as early as E15 show that axons form a highly ordered array, reflecting the spatiotemporal wave of maturation that sweeps across the developing cortex. Moreover, this array fills the intermediate zone, through which thalamic axons subsequently grow in the opposite direction as a similar topographic array. Back-labeling from the internal capsule at this stage (Fig. 7) shows that cells in both divisions of the original preplatemarginal zone as well as subplate - contribute to the early corticofugal axon array.

Corresponding thalamic and cortical axons enter the lateral part of the internal capsule approximately simultaneously, from opposite sides (McConnell et al., 1989; Molnár and Blakemore, 1990; Bicknese and Pearlman, 1992; De Carlos and O'Leary 1992; Erzurumlu and Jhaveri, 1992; Molnár et al., 1998). Preplate axons could constitute a scaffold (McConnell et al., 1989) over which thalamocortical fibers grow toward the appropriate cortical area-the handshake hypothesis (Molnár and Blakemore, 1995).

The most direct test of the handshake hypothesis is to use double labeling to see whether axons from corresponding cortical and thalamic regions do meet and intermingle. There is a narrow window of opportunity, when the two axon systems can indubitably be separately labeled from the cortex and the thalamus (E14.5 to early E16 for the geniculocortical pathway). But, paradoxically, it is impossible to be certain of the precise spatial correspondence between thalamic and cortical regions until thalamic axons actually reach their target areas.

Miller et al. (1993) did not see obviously intermixed thalamic and cortical axons in any of their nine double-labeling experiments in the hamster. But the handshake hypothesis predicts that even slight offset in double labeling should result in nonoverlapping fiber bundles, so negative results are not definitive. We too saw little or no overlap in $42 \%$ of 26 such experiments between E14.5 and E15.5 in the rat. However, in $58 \%$ of cases, substantial fractions of the axon bundles were clearly mixed, with thalamic and preplate fibers juxtaposed, following the same trajectories, even in thin confocal optical sections and in stereo reconstructions (Fig. 10). Ascending and descending axon systems, at least for the dorsolateral segment of the cortex, share the same compartment within the internal capsule and the intermediate zone. Bicknese and Pearlman (1992) and Erzurumlu and Jhaveri (1992), using double labeling in the mouse and the hamster, also reported that thalamic and early corticofugal fibers interdigitate in the internal capsule.

The full handshake hypothesis proposes that thalamic fibers navigate all the way to the cortex along the axons of subplate cells. However, Bicknese et al. (1994) described thalamic axons in the rat extending within the subplate layer, whereas fibers of subplate and cortical plate cells ran below the subplate. On the basis of these observations, they suggested that ascending and descending axons run in separate, adjacent compartments close to the target area. On the other hand, labeling with small crystals in the internal capsule between E15 and E16 clearly shows a broad array 
of identifiable thalamic axons running in close association with corticofugal fibers, right up to the subplate (Figs. 11, 12). These findings, together with the positive results of double labeling, strongly suggest that many thalamic fibers follow preplate axons right to the target area. Although some thalamic axons may continue to grow further within the subplate layer, the two projection systems appear to share the same growth compartment and to run along similar trajectories within the intermediate zone, at least toward the dorsolateral segment of the cortex.

Further powerful evidence that descending and ascending arrays are at least approximately locked in correspondence comes from the arrangement of individual, discrete bundles of mixed afferent and efferent axons seen after multiple crystal placements in the cortex at E16 (Fig. 14D). The results of similar experiments at later ages, when the axons of cells of layers 6 and 5 are also labeled, suggest that they follow the same basic global topography as the earlier projections, until the point at which layer 5 axons peel off at the medial edge of the internal capsule to form the cerebral peduncle (Fig. 15). Thus the pioneering preplate scaffold may play some part in guiding not only afferent thalamic axons but also other descending corticofugal projections, as suggested by McConnell et al. (1989).

In the adult rat, corticofugal axons from the dorsal occipital (visual) cortex run some $150 \mu \mathrm{m}$ deep to the corresponding thalamocortical axons in the white matter (Woodward and Coull, 1984; Woodward et al., 1990). Perhaps postnatal loss of subplate axons leads to the appearance of this distinct misalignment of afferent and efferent fibers in the adult.

Of course, even close association of corresponding preplate and thalamic axons does not prove that the former actually guide the latter. But, at the very least, the early corticof ugal scaffold and the subplate layer appear to provide attractive compartments within which thalamic axons grow. The subplate is rich in chondroitin sulfate proteoglycans (Bicknese et al., 1994), especially neurocan (Fukuda et al., 1997), and it is possible that proteoglycan expression in the intermediate zone, although much weaker (Bicknese and Pearlman, 1992; Fukuda et al., 1997), is associated with the axons of subplate cells. Indeed, Emerling and Lander (1994) found that dissociated embryonic thalamic neurons selectively adhere to and extend neurites on the intermediate zone as well as the subplate of embryonic cortical slices (E15-E16), and this behavior is eliminated by the enzymatic removal of chondroitin sulfate (Emerling and Lander, 1996).

\section{Is there a "waiting period" in rodents?}

Although the accumulation of thalamic fibers below the cortical plate, which is such a striking feature of development in carnivores (Shatz and Luskin, 1986; Ghosh and Shatz, 1992) and primates (Rakic, 1977, Kostovic and Rakic, 1990), was first described by Lund and Mustari (1977) in the rat, the existence of a waiting period in rodents has recently been questioned. Catalano et al. (1991, 1996) and Kageyama and Robertson (1993) observed thalamic fibers simply advancing steadily into the cortex as it matures. We saw some axons entering the lower layers of the occipital cortex as early as E16 (Fig. 5), but they were rare and trivial in density compared with the massive invasion that starts at E19-E20, 2-3 d after their arrival (Figs. 3, 5). This is compatible with the results of co-culture experiments, which suggest that the occipital cortex does not express membrane-bound growthpermissive properties until approximately E19-E20 (Götz et al., 1992; Molnár and Blakemore, 1995).

\section{Chemospecificity and chronotopy}

Molecular signals, perhaps expressed in gradients across the developing cortex, might play a part in guiding, or at least refining, the distribution of thalamic fibers. Experiments involving coculture of thalamic and cortical explants (Yamamoto et al., 1992) suggest that the cortex produces a diffusible factor that stimulates the outgrowth of thalamic neurites from as early as E14 (Blakemore and Molnár, 1990; Molnár and Blakemore, 1991, 1995; Rennie et al., 1994; Lotto and Price 1995; Molnár, 1998). This remote influence may play a part in initiating outgrowth of thalamic axons in vivo, but it seems inconceivable that it could impose the topographic order that they exhibit throughout their course.

Bolz and Götz (1992) have shown that a thalamic explant extends neurites somewhat more profusely over a surface coated with membranes prepared from the corresponding region of cortex than over a membrane preparation from the opposite end of the hemisphere. However, the cortex does not express membrane-bound growth-permissive properties until after thalamic axons have already arrived, in topographic order, below the cortical plate; it therefore seems unlikely that such properties control their overall distribution. However, chemical cues expressed at the growing tips of preplate axons might play a role in establishing their relationships with thalamic axons during the handshake.

In the rat, like all mammalian species so far examined, there is a pronounced gradient of maturation across the cerebral hemispheres (Bayer and Altman, 1991). There is also a temporal pattern of maturation within the thalamus (Jones, 1985), the first thalamic axons to reach the cortex being from the ventrobasal complex (Braisted and O'Leary, 1995). If outgrowing axons deposit themselves on the carpet of axons that have already extended, and if axons tend to maintain neighborly order and not to cross each other, the timing of fiber outgrowth could potentially determine one axis of the initial mapping of thalamus to cortex (Molnár and Blakemore, 1995).

\section{REFERENCES}

Agmon A, Connors BW (1991) Thalamocortical responses of mouse somatosensory (barrel) cortex in vitro. Neuroscience 41:365-379.

Agmon A, Yang LT, O'Dowd DK, Jones EG (1993) Organized growth of thalamocortical axons from the deep tier of terminations into layer IV of mouse barrel cortex. J Neurosci 13:5365-5382.

Agmon A, Yang LT, Jones EG, O'Dowd DK (1995) Topological precision in the thalamic projection to neonatal mouse barrel cortex. J Neurosci 15:549-561.

Bayer SA, Altman J (1991) Neocortical development. New York: Raven.

Bernardo KL, Woolsey TA (1987) Axonal trajectories between mouse somatosensory thalamus and cortex. J Comp Neurol 258:542-564.

Bicknese AR, Pearlman AL (1992) Growing corticothalamic and thalamocortical axons interdigitate in a restricted portion of the forming internal capsule. Soc Neurosci Abstr 18:778.

Bicknese AR, Sheppard AM, O'Leary DDM, Pearlman AL (1994) Thalamocortical axons extend along a chondroitin sulfate proteoglycan-enriched pathway coincident with the neocortical subplate and distinct from the efferent path. J Neurosci 14:3500-3510.

Blakemore C, Molnár Z (1990) Factors involved in the establishment of specific interconnections between thalamus and cerebral cortex. Cold Spring Harb Symp Quant Biol 55:491-504.

Bolz J, Götz M (1992) Mechanisms to establish specific thalamocortical connections in the developing brain. In: Development of the central nervous system in vertebrates (Sharma SC, Goffinet AM, eds), pp 179-192. New York: Plenum.

Braisted JE, O'Leary DDM (1995) Axons from the ventrobasal thalamic nucleus pioneer the thalamocortical pathway to rat neocortex. Soc Neurosci Abstr 21:798.

Brodmann K (1909) Vergleichende Localisationslehre der Grosshirn- 
rinde in ihren Prinzipien dargestellt auf Grund des Zellenbaues. Leipzig, Germany: Barth.

Catalano S, Robertson RT, Killackey HP (1991) Early ingrowth of thalamocortical afferents to the neocortex of the prenatal rat. Proc Natl Acad Sci USA 88:2999-3003.

Catalano S, Robertson RT, Killackey H (1996) Individual axon morphology and thalamocortical topography in developing rat somatosensory cortex. J Comp Neurol 366:36-53.

Caviness Jr VS (1988) Architecture and development of the thalamocortical projection in the mouse. In: Cellular thalamic mechanisms (Bentivoglio M, Spreafico R, eds), pp 489-499. Amsterdam: Elsevier.

Caviness Jr VS, Frost DO (1980) Tangential organization of thalamic projections of the neocortex in the mouse. J Comp Neurol 194:355-367.

Clasca F, Angelucci A, Sur M (1995) Layer-specific programs of development in neocortical projection neurons. Proc Natl Acad Sci USA 92:11145-11149.

Crandall JE, Caviness Jr VS (1984) Axon strata of the cerebral wall in the embryonic mice. Dev Brain Res 14:185-195.

De Carlos JA, O'Leary DDM (1992) Growth and targeting of subplate axons and establishment of major cortical pathways. J Neurosci 12:1194-1211.

Emerling DE, Lander AD (1994) Laminar specific attachment and neurite outgrowth of thalamic neurons on cultured slices of developing cerebral neocortex. Development 120:2811-2822.

Emerling DE, Lander AD (1996) Inhibitors and promoters of thalamic neuron adhesion and outgrowth in embryonic neocortex: functional association with chondroitin sulfate. Neuron 17:1089-1100.

Erzurumlu RS, Jhaveri S (1992) Emergence of connectivity in the embryonic rat parietal cortex. Cereb Cortex 2:336-352.

Frost DO, Caviness Jr VS (1980) Radial organization of thalamic projections to the neocortex in the mouse. J Comp Neurol 194:369-393.

Fukuda T, Kawano H, Ohyama K, Li H-P, Takeda Y, Oohira A, Kawamura K (1997) Immunohistochemical localization of neurocan and $\mathrm{L} 1$ in the formation of thalamocortical pathway of developing rats. J Comp Neurol 382:141-152.

Ghosh A, Shatz CJ (1992) Pathfinding and target selection by developing geniculocortical axons. J Neurosci 12:39-55.

Godement P, Vanselow J, Thanos S, Bonhoeffer F (1987) A study in developing visual systems with a new method of staining neurones and their processes in fixed tissue. Development 101:697-713.

Götz M, Novak N, Bastmeyer M, Bolz J (1992) Membrane bound molecules in the rat cerebral cortex regulate thalamic innervation. Development 116:507-519.

Jones EG (1985) The thalamus. New York: Plenum.

Johnson GD, Davidson RS, McNamee KC, Russell G, Goodwin D, Holbarow EJ (1982) Fading of immunofluorescence during microscopy: a study of the phenomenon and its remedy. J Immunol Methods 55:231-242.

Kageyama GH, Robertson RT (1993) Development of geniculocortical projections to visual cortex in rat: evidence for early ingrowth and synaptogenesis. J Comp Neurol 335:123-148.

Kostovic I, Rakic P (1990) Developmental history of the transient subplate zone in the visual and somatosensory cortex of the macaque monkey and human brain. J Comp Neurol 297:441-470.

Lotto RB, Price DJ (1995) The stimulation of thalamic neurite outgrowth by cortical-derived growth factors in vitro: the influence of cortical age and activity. Eur J Neurosci 7:318-328.

Lund RD, Mustari MJ (1977) Development of the geniculocortical pathway in rats. J Comp Neurol 173:289-305.

Luskin MB, Shatz CJ (1985a) Neurogenesis of the cat's primary visual cortex. J Comp Neurol 242:611-631.

Luskin MB, Shatz CJ (1985b) Studies of the earliest-generated cells of the cat's visual cortex: cogeneration of subplate and marginal zones. J Neurosci 5:1062-1075.

Marin-Padilla M (1971) Early prenatal ontogenesis of the cerebral cor- tex (neocortex) of the cat (Felis domestica): a Golgi study. I. The primordial neocortical organization, $\mathrm{Z}$ Anat Entwicklungsgesch 134:117-145.

McConnell SK, Gosh A, Shatz CJ (1989) Subplate neurons pioneer the first axon pathway from the cerebral cortex. Science 245:978-982.

Métin C, Godement P (1996) The ganglionic eminence may be an intermediate target for corticofugal and thalamocortical axons. J Neurosci 16:3219-3235.

Miller B, Chou L, Finlay BL (1993) The early development of thalamocortical and corticothalamic projections. J Comp Neurol 335:16-41.

Miller MW (1988) Development of projection and local circuit neurons in neocortex. In: Cerebral cortex, Vol 7 (Peters A, Jones EG, eds), pp 133-175. New York: Plenum.

Mitrofanis J (1992) Patterns of antigenic expression in the thalamic reticular nucleus of developing rats. J Comp Neurol 320:161-181.

Mitrofanis J, Baker GE (1993) Development of the thalamic reticular and perireticular nuclei in rats and their relationship to the course of growing corticofugal and corticopetal axons. J Comp Neurol 338:575-587.

Mitrofanis J, Guillery RW (1993) New views of the thalamic reticular nucleus in the adult and developing brain. Trends Neurosci 16:240-245.

Molnár Z (1998) Development of thalamocortical connections. Neuroscience Intelligence Unit Series. Austin, TX: Landes.

Molnár Z, Blakemore C (1990) Relationship of corticofugal and corticopetal projections in the prenatal establishment of projections from thalamic nuclei to the specific cortical areas of the rat. J Physiol (Lond) 430:104P.

Molnár Z, Blakemore C (1991) Lack of regional specificity for connections formed between thalamus and cortex in co-culture. Nature 351:475-477.

Molnár Z, Blakemore C (1995) How do thalamic axons find their way to the cortex? Trends Neurosci 18:389-397.

Molnár Z, Adams R, Goffinet A, Blakemore C (1998) The role of the first postmitotic cortical cells in the development of thalamocortical innervation in the reeler mouse. J Neurosci 18:5746-5765.

Naegele JR, Jhaveri S, Schneider GE (1988) Sharpening of topographical projections and maturation of geniculocortical axon arbors in the hamster. J Comp Neurol 277:593-607.

O'Leary DDM (1989) Do cortical areas emerge from a protocortex? Trends Neurosci 12:400-406.

Peters A, Jones EG (1985) Cerebral cortex, Vols 3 and 4. New York: Plenum.

Rakic P (1977) Prenatal development of the visual system in the rhesus monkey. Philos Trans R Soc Lond B Biol Sci 278:245-260.

Rennie S, Lotto RB, Price DJ (1994) Growth-promoting interactions between the murine neocortex and thalamus in organotypic co-cultures. Neuroscience 61:547-564.

Shatz CJ (1990) Impulse activity and the patterning of connections during CNS development. Neuron 5:745-756.

Shatz CJ, Luskin MB (1986) Relationship between the geniculocortical afferents and their cortical target cells during development of the cat's primary visual cortex. J Neurosci 6:3655-3668.

Wise SP, Jones EG (1978) Developmental studies of thalamocortical and commissural connections in the rat somatic sensory cortex. J Comp Neurol 178:187-208.

Woodward WR, Coull BM (1984) Localisation and organisation of geniculocortical and corticofugal fiber tracts within the subcortical white matter. Neuroscience 12:1089-1099.

Woodward WR, Chiaia N, Teyler TJ, Leong L, Coull BM (1990) Organization of cortical afferent and efferent pathways in the white matter of the rat visual system. Neuroscience 36:393-401.

Yamamoto N, Yamada K, Kurotani T, Toyama K (1992) Laminar specificity of extrinsic cortical connections studied in culture preparations. Neuron 9:217-228. 